\title{
LA DIVERSIDAD LOCAL FRENTE A LA «CULTURA CHACHAPOYA» EN LA ARQUITECTURA DOMÉSTICA
}

\author{
Anna Guengerich ${ }^{\mathrm{a}}$
}

\begin{abstract}
Resumen
Este artículo critica la idea que las sociedades preinka de Chachapoyas compartieran una tradición de arquitectura doméstica que las distinguieran de las regiones vecinas. Con frecuencia, la presencia de una supuesta «arquitectura chachapoya" en un contexto arqueológico se toma como indicador de tales sociedades. Como resultado, el concepto ha adquirido importancia notable en una situación actual de estudios regionales, en la que se vienen acumulando evidencias de que existió gran variación a nivel local, en cuanto a otros campos de cultura material, como la alfarería fina y los entierros en barrancos. En cuanto a la arquitectura doméstica, tres rasgos -las cornisas, los frisos y las bases plataforma - son interpretados con frecuencia como típicos de la tradición «chachapoya». Pero la comparación de varias subregiones principales —entre ellos Luya, los distritos de Leymebamba y Chuquibamba y la cuenca del Sonche-, devela gran variabilidad de los atributos arquitectónicos, y en algunos sitios, ni siquiera se encuentran estos tres rasgos, supuestamente tipicos de la arquitectura "chachapoya". En contraste con las otras formas de cultural material, donde si se reconocen las variaciones locales, la tendencia a suponer una homogeneidad en el campo de la arquitectura doméstica refleja marcos teóricos ampliamente difundidos en la arqueología. El reconocimiento de la diversidad arquitectónica que existió en Chachapoyas antes de la llegada de los incas promete enriquecer nuestras visiones actuales de la diversidad social y cultural que caracterizó a esta región y ayudará a problematizar la construcción cultural y analítica de concepto de "Chachapoyas» en el presente y el pasado.
\end{abstract}

Palabras clave: arquitectura doméstica, diversidad, Chachapoyas

\section{Abstract}

\section{THE LOCAL DIVERSITY VERSUS «CHACHAPOYA CULTURE» IN DOMESTIC ARCHITECTURE}

This article critiques the notion that pre-Inka societies across Chachapoyas shared a common tradition of domestic architecture that distinguished them from neighboring regions. This notion frequently appears in regional scholarship as a means by which to identify the presence of culturally "Chachapoya" groups, and it has gained special prominence in light of mounting evidence that considerable local variation existed in other forms of material culture in Chachapoyas, such as fineware ceramics and cliff-tomb burials. In terms of domestic architecture, three features in particular-cornices, friezes, and platform bases—are often cited as typifying a "Chachapoya" architectural tradition. Yet a detailed comparison of studies from several major sub-regions-including Luya, the Leymebamba-Chuquibamba districts, and the Sonche Basin — reveals considerable variation in architectural attributes. Indeed, in some areas the typical "Chachapoya" features are absent altogether. In contrast to other forms of material culture in which local variations are, in fact, recognized, the tendency to perceive a supposed homogeneity of domestic architecture reflects common theoretical frameworks in archaeology. Recognizing the local architectural diversity present in Chachapoyas, however, promises to enrich contemporary understandings of the cultural and social diversity that characterized this region prior to Inka conquest, and will help problematize the cultural and analytical construction of the concept of "Chachapoyas» in the past and present.

Keywords: domestic architecture, diversity, Chachapoyas

${ }^{\text {a }}$ Department of Anthropology, Vanderbilt University

Correo electrónico: anna.guengerich@vanderbilt.edu 


\section{Introducción}

La arquitectura doméstica, tanto para los arqueólogos como para el público general, es una de las formas de cultura material más representativa de la región arqueológica de Chachapoyas ${ }^{1}$. La vivienda circular, construida en piedra con frisos geométricos y techo cónico de paja (Fig. 4.3), es un símbolo principal de esta región tanto a nivel local como al nivel nacional. Este rol se refleja en su presencia ampliamente visible en los afiches turísticos, las filmaciones, la artesanía y hasta los llaveros encontrados igualmente en las tiendas de Amazonas y las empresas de viaje de Lima y Cusco (Fig.1).

En años recientes, la arquitectura circular de piedra ha adquirido un significado adicional para los estudiosos como indicador de las sociedades pertenecientes a la llamada "cultura chachapoya». Este interés en la arquitectura circular se puede atribuir en parte al aumento en las últimas décadas del interés en la arqueología de Chachapoyas, el cual viene acompańado por el deseo de desarrollar maneras de identificar sistemáticamente a la presencia de tales sociedades. Diversos autores (v.g. Schjellerup 2005: 449; Church y von Hagen 2008: 913; Koschmieder y Gaither 2010: 11; Ruiz Estrada 2013: 3) han planteado que la arquitectura doméstica constituyó una tradición material que integrara esta inmensa región y la distinguía de las regiones vecinas de los Andes y la Amazonía. Tres rasgos en particular: las cornisas, las bases plataforma y, sobre todo, los frisos, sobresalen por su asociación con la región arqueológica de Chachapoyas. Varios investigadores, pues, los han considerado parte de un estilo arquitectónico que diferencia a estos grupos de sus contemporáneos andinos y amazónicos (v.g. Church 1994: 283; von Hagen 2002a: 92; Schjellerup 2005: 203). En algunos casos, incluso en zonas tan alejadas como Lamas y la provincia de Bagua (v.g. Narváez 2013: 115-116), los investigadores han postulado una presencia "chachapoya» solo sobre la base de la presencia de estructuras circulares de piedra.

Tal interés en la arquitectura doméstica también debe interpretarse en el contexto de una tendencia más amplia en los estudios regionales, en que los investigadores van reconociendo la gran variación que existió al nivel local ${ }^{2}$ en varios campos de cultural material (Guengerich y Church, este volumen). Desde hace décadas, los investigadores han postulado que las diferencias entre las prácticas funerarias - las chullpas versus los purunmachus, o saracófagos de barro-, distinguían a la margen derecha y la izquierda del río Utcubamba (Horkheimer 1959; Crandall 2012; Kauffman y Ligabue 2003). En obras recientes, Arturo Ruiz Estrada (2011 y este número) y Klaus Koschmieder (2012, 2014, 2015), han llamado atención sobre la identidad sociopolítica distinta de los habitantes de Luya y Chillaos, desde perspectivas etnohistóricas y arqueológicas. Koschmieder (2015), subraya las prácticas en las que los de Luya se diferenciaban de otras partes de Chachapoyas, como por ejemplo la dieta y la cerámica fina: el maíz y la cerámica pintada dominaban en Luya, mientras que los tubérculos y la cerámica modelada dominaban a las zonas más altas de la cuenca superior del Utcubamba y el Atuén. Otras investigaciones recientes y en marcha en varias zonas más allá del río Utcubamba — el cual se ha reconocido históricamente como el corazón de Chachapoyas-, prometen brindar aún más ejemplos de la variación en la cultura material de la extensa región de Chachapoyas (v.g. Fabre et al. 2008; Bueno y Cornejo 2009; Schjellerup et al. 2003, 2005, 2009; Risco y Crandall en prep.; Church y Valle este número; McCray, este número). Sobre la base de estas diferencias materiales, así como de fuentes etnohistóricas, varios investigadores vienen cuestionando la existencia de una "cultura chachapoya», que uniera supuestamente a todas las sociedades preinka de la región arqueológica ahora conocida como «Chachapoyas» (Ruiz Estrada 2011; Koschmieder 2012). Lo irónico es que fue un rasgo arquitectónico — los frisos— unas de las primeras formas de cultura material en la que se reconoció la presencia de estas variaciones locales (v.g. Lerche 1986, Schjellerup 2005[1997]).

A pesar de tal reconocimiento, los estudios actuales siguen reforzando la idea de que la arquitectura doméstica haya formado un substrato cultural fundamental que uniera a las diversas zonas geográficas y sociedades locales de Chachapoyas. En este artículo se revisan las evidencias materiales de esta idea y propongo que esta idea se ha sostenido en el tiempo debido a la escasez de datos 


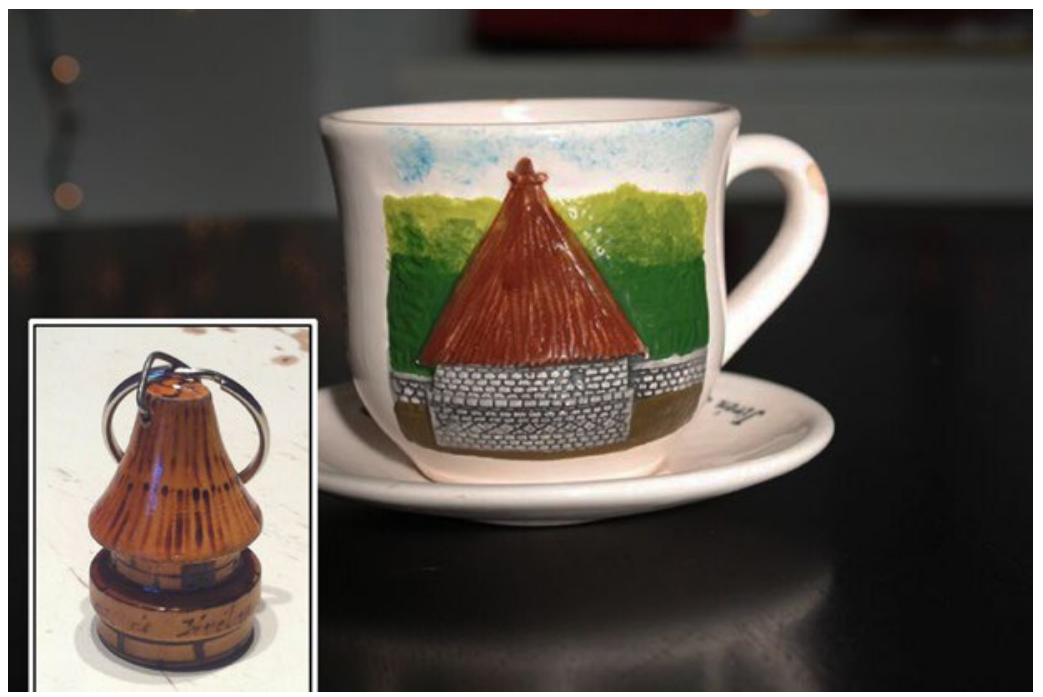

Figura 1. La "choza chachapoya» circular representada en la imaginación popular: un llavero y una taza emblemático de un restaurante en la ciudad de Chachapoyas (foto por A. Guengerich).

arqueológicos, la falta de comparaciones sistemáticas entre distintas localidades y una tendencia a tener una visión más general de esta región. En realidad, la inspección minuciosa de la arquitectura doméstica demuestra diferencias notables al nivel local al igual que sucede en otros aspectos materiales, lo cual apoya la conclusión de que no existió una sola «cultural chachapoya» que integrara a toda esta inmensa región.

\section{2. ¿Cómo se reconoce «la arquitectura chachapoya»?}

Empezaremos con una revisión de las investigaciones que se han llevado a cabo hasta la fecha, las cuales han establecido las características encontradas con más frecuencia en la arquitectura preinka de esta región. Sobre la base de estas características, se ha "producido» la imagen clásica de la "casa chachapoya", conocida tanto en la literatura arqueológica como en los afiches turísticos. Consideraremos también la cronología y los rasgos descritos con más frecuencia, además de revisar los ejes de variación que sí se han identificado hasta ahora.

\subsection{La cronología y el desarrollo}

Las tradiciones de arquitectura doméstica que se encontraban en Chachapoyas al momento de la llegada de los inkas no siempre habían estado presentes en esta región, sino que parecen haberse desarrollado en un momento particular a fines del Horizonte Medio o principados del Período Intermedio Tardío. Las fechas más tempranas asociadas a un edificio circular ser remontan a un poco antes de 1000 d.C., siendo el ejemplo más temprano la Estructura 51 de Monte Viudo, la cual fue fechada en 861-1018 d.C. (calib. SHCal13) (Guengerich 2014a). La tradición de la arquitectura circular de piedra duró más de quinientos años, aunque es difícil saber cuándo se desvaneció debido a la continuación de su uso bajo el dominio Inka y tal vez después. Aun en el siglo XIX, los viajeros Arthur Werthemann (1892), Charles Wiener (1894) y Louis Langlois (1939) describieron una vivienda circular que seguía en uso en la ciudad de La Jalca (Fig. 2).

Por otro lado, no sabemos mucho de las características de la arquitectura que existió en esta región antes del Período Intermedio Tardío. En el sitio de Tosán, en Luya, Koschmieder (2012: 37) encontró huecos de poste de madera en excavaciones asociadas a fechas de 370-535 d.C. A partir 

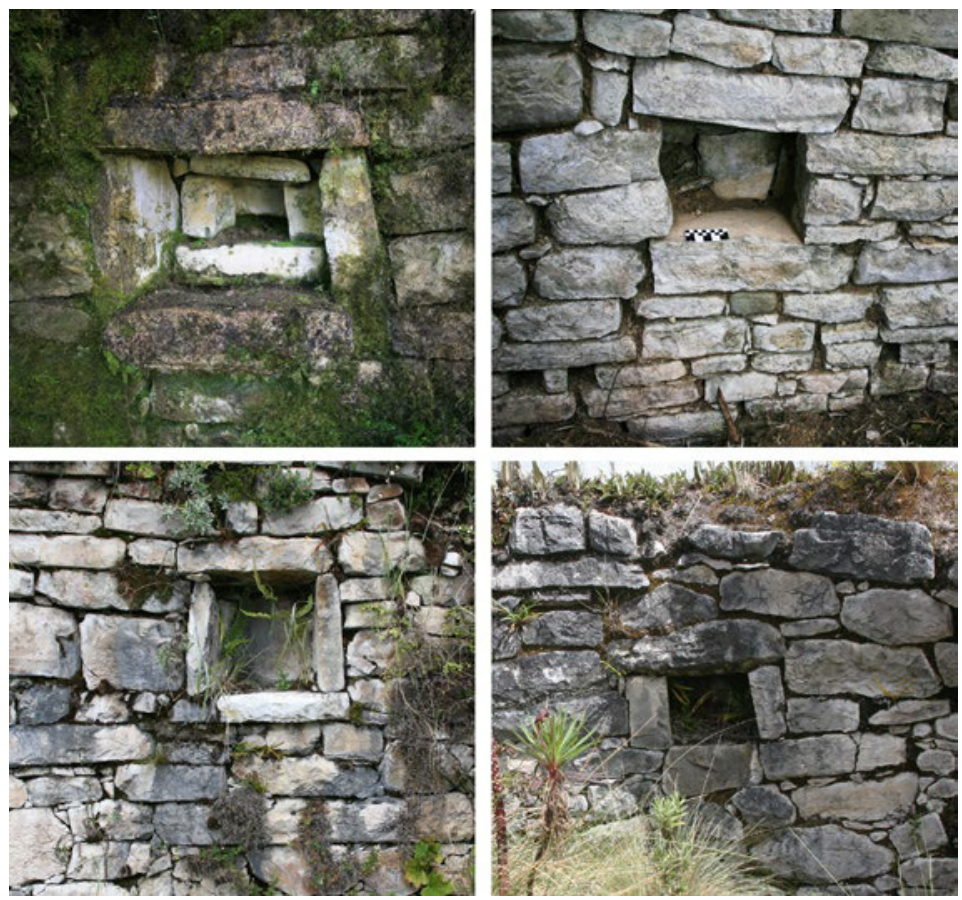

Figura 2. Ejemplares de hornacinas encontradas en Chachapoyas: (empezando en el lado izquierda, superior) de La Joya, Monte Viudo (Estructura 192), La Vaquería, y Bóveda (foto por A. Guengerich).

de estos datos, postula que antes del desarrollo de la arquitectura de piedra, existió una tradición de arquitectura de materiales perecederos, como la quincha. Las excavaciones en Monte Viudo, en la cuenca del río Atuén, también revelaron rasgos que posiblemente representan huecos de poste. Estos fechaban en 391 a.C.-110 d.C. (Guengerich 2014a: 97), unos siglos antes de los huecos de postes descritos por Koschmieder. Aunque son pocas estas evidencias, la falta total de vestigios arquitectónicos de piedra en Chachapoyas con fechas antes del siglo IX, refuerza la interpretación de que las tradiciones arquitectónicas regionales experimentaron un gran cambio a fines del primer milenio d.C. Desafortunadamente, la falta general de investigaciones que han tratado esta época de la historia regional hace difícil interpretar el significado de estos cambios.

Después de 1000 d.C., se desarrollaron dos características que se encontraban en las arquitecturas locales de todo Chachapoyas (Schjellerup 2005; Fabre 2006; Church y von Hagen 2008; Guengerich 2014b; Koschmieder 2015). Primero, la piedra empezó a utilizarse como el principal material constructivo para los muros y los basamentos. Segundo, en todos los sitios de la región, la gran mayoría de los edificios fueron construidos según un plano circular, o a veces con forma ligeramente ovoide o irregular de acuerdo con el terreno. Generalmente se caracterizaban por diámetros internos de 4 a 6 metros, pero también existen ejemplos de construcciones circulares con diámetros de 10 metro a más (v.g. Narváez Vargas 1996a; Koschmieder 2014), las cuales posiblemente sirvieron para otros propósitos diferentes de las actividades domésticas.

También existió una gama de rasgos arquitectónicos que se encuentra en muchos sitios y muchas áreas locales, aunque no todos. Frecuentemente, la mampostería de los muros incorporaba la roca madre, mostrando una técnica y un aspecto estético mejor conocido en la arquitectura inka ( $c f$. Dean 2010). En casi todos los asentamientos, se encuentran viviendas con hornacinas en las paredes interiores, que tenían forma rectangular, cuadrada, o ligeramente trapezoidal (Fig. 3). También se encuentra con frecuencia una serie de huecos cuadrados de aproximadamente 8 por 8 centímetros, que trazan una línea horizontal sobre la pared interior, a un metro o más sobre el nivel del piso 


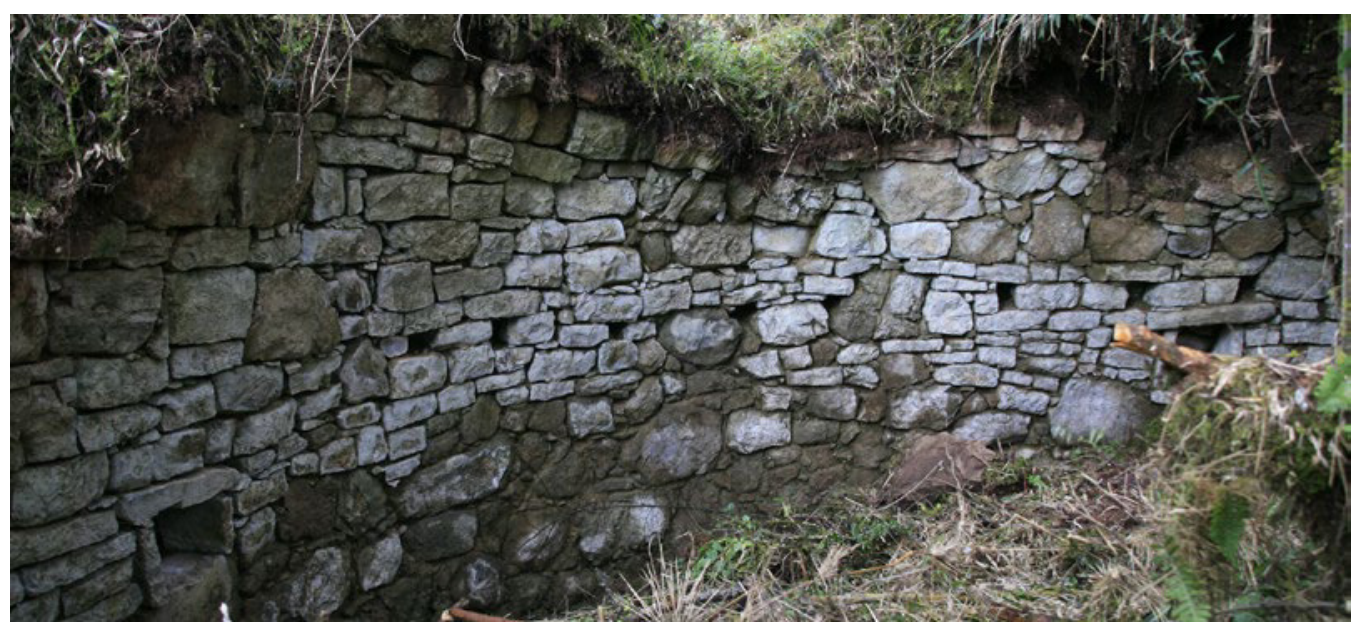

Figura 3. Un alineamiento de huecos cuadrados al fondo interior de Estructura 192, en Monte Viudo (foto por A. Guengerich).
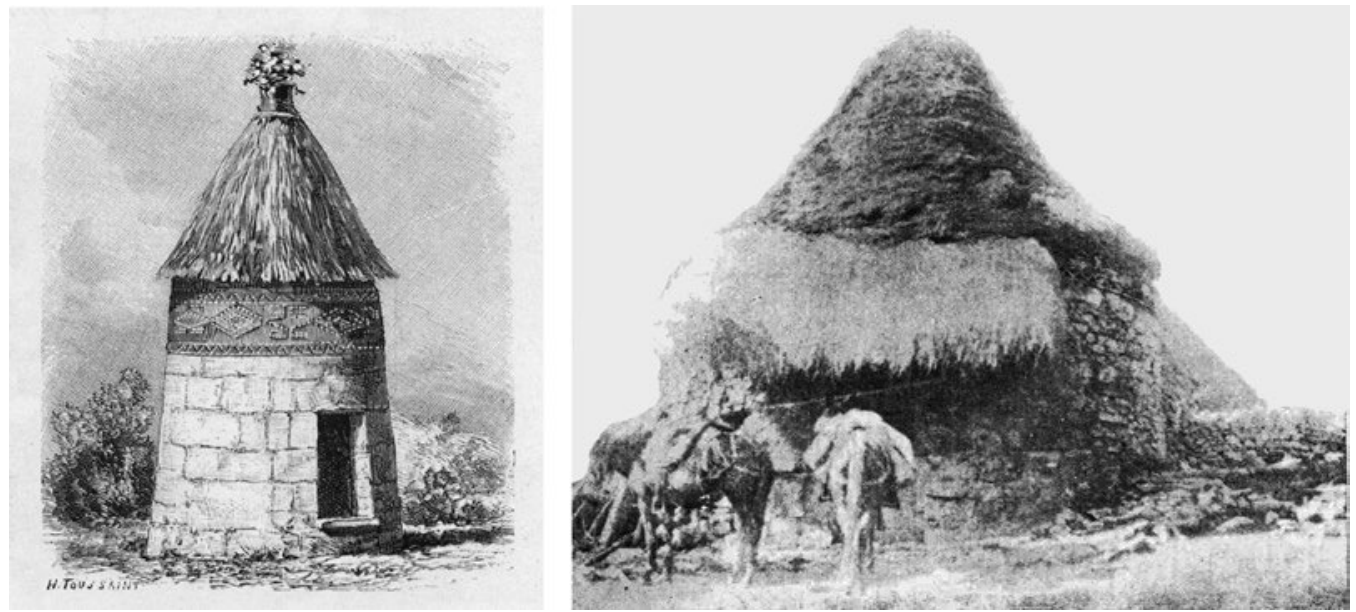

Figura 4. Dibujo y fotografia del último edificio circular habitado en Chachapoyas, en la ciudad de La Jalca. De Werthemann (1892) (izquierda) y Langlois (1940) (derecha).

(Fig. 4). Se ha propuesto que sostuvieran vigas para colgar utensilios domésticos, o que apoyaban un recinto superior o el techo mismo (Langlois 1939; Narváez Vargas 1996; Schjellerup 2005: 360; Guengerich 2014a). En cuanto a los techos, generalmente ha sido aceptado el modelo de Morgan Davis (1996), quien utilizó las descripciones de los viajeros Charles Wiener (1894) y Louis Langlois (1939) sobre la última estructura circular en uso continuo a fines del siglo XIX (Fig. 2) para reconstruir una estructura circular con techo cónico de paja y madera en el pueblo de Colla Cruz.

\subsection{Una tríada arquitectónica: las bases plataforma, las cornisas y los frisos}

Entre todos los rasgos que caracterizaban a la arquitectura circular, sobresalen tres que se limitaban casi exclusivamente a Chachapoyas: las bases plataforma, las cornisas y los frisos. Las «bases plataforma» consistieron en plataformas de piedra que elevaban los pisos de las viviendas (o sea la «sobreestructura») sobre la superficie del suelo (Fig. 5). Aunque esto le brinda a la estructura un 


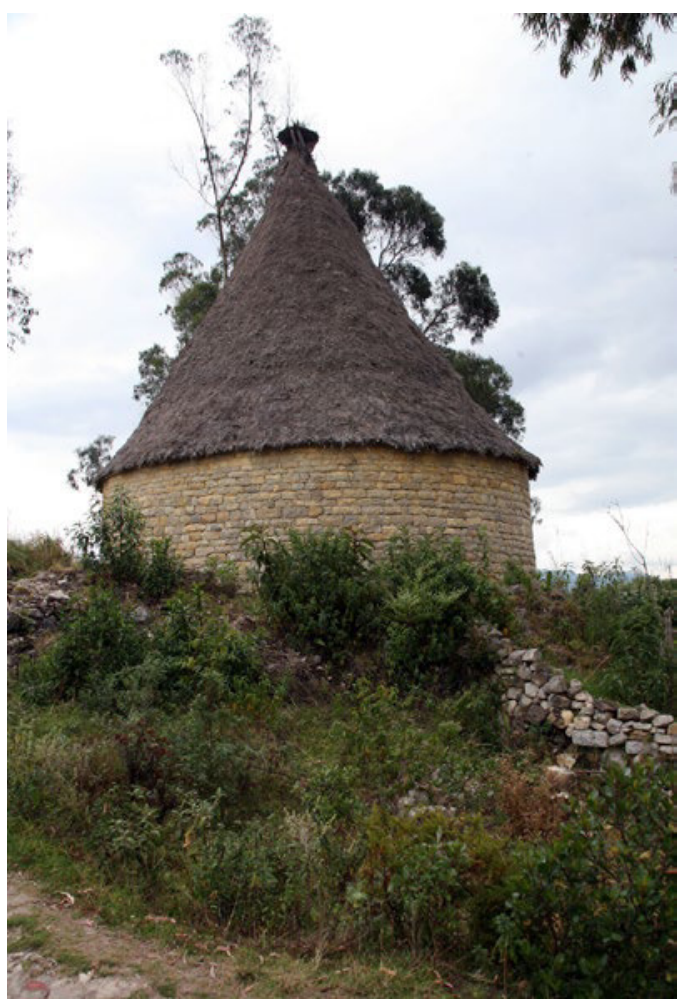

Figura 5. Edificio circular de Colla Cruz reconstruido por Davis (foto por A. Guengerich).

aspecto de tener dos pisos, en realidad las bases-plataforma son construcciones sólidas con relleno de tierra y piedras sueltas. La gran mayoría de las bases plataforma tenía forma circular, del mismo diámetro de la "sobre-estructura» o ligeramente más grande, pero también existen bases cuadrangulares (v.g. Fabre 2006; Alexandrino y Guengerich 2017) (Fig. 5, derecha). Las bases plataforma cumplieron varias funciones. Primero, posibilitaron la construcción en terreno accidentado y rocoso, donde se encuentran la mayoría de los asentamientos en Chachapoyas. En otras regiones serranas del Período Intermedio Tardío (v.g. Bonnier 1997; Arkush 2005), esto se consiguió mediante la construcción de terrazas domésticas, que fueron compartidas entre varias viviendas y probablemente fueron utilizadas por grupos familiares. Tales tipos de terrazas también se encuentran en Chachapoyas (v.g. Narváez Vargas 1996; Schjellerup 2005; Ruíz Barcellos 2007; Fabre et al. 2008; Koschmieder 2015), incluso en los mismos sitios donde también se encuentran edificios circulares con bases plataforma. Pero las bases plataformas también parecen haber cumplido el rol social de expresar o aumentar el estatus o el prestigio del grupo que construyó la vivienda, o que allí vivía. Por ejemplo, en Monte Viudo, las bases de las viviendas en el Sector Oeste, un barrio cuyos rasgos arquitectónicos reflejan la posición social elevada de los habitantes (Guengerich 2014a), llegaban hasta 3 metros de altura - más grandes de lo necesario para adaptarse al terreno (Fig. 5, centro). En algunos casos, como en Kuélap (Narváez Vargas 1996), se edificaron bases plataforma en terrenos artificialmente nivelados, donde estas no servían a ningún propósito técnico. Las bases plataforma nunca se presentan en todos los edificios de un mismo sitio, por lo que sería difícil categorizarlas como un componente integral de un estilo regional vernáculo. Por el contrario, su presencia parece reflejar una variedad de factores sociales y ambientales.

Por su parte, las cornisas, o «aleros», se usaban siempre en combinación con la base plataforma. Estas consisten en un anillo horizontal de lajas sobresalientes en forma de halo que se ubica en la pared exterior donde se juntan la base y la parte superior del edificio (Fig. 6). Como las bases plataforma, las cornisas cumplían varias funciones. A nivel técnico, funcionaban como pasarela que 

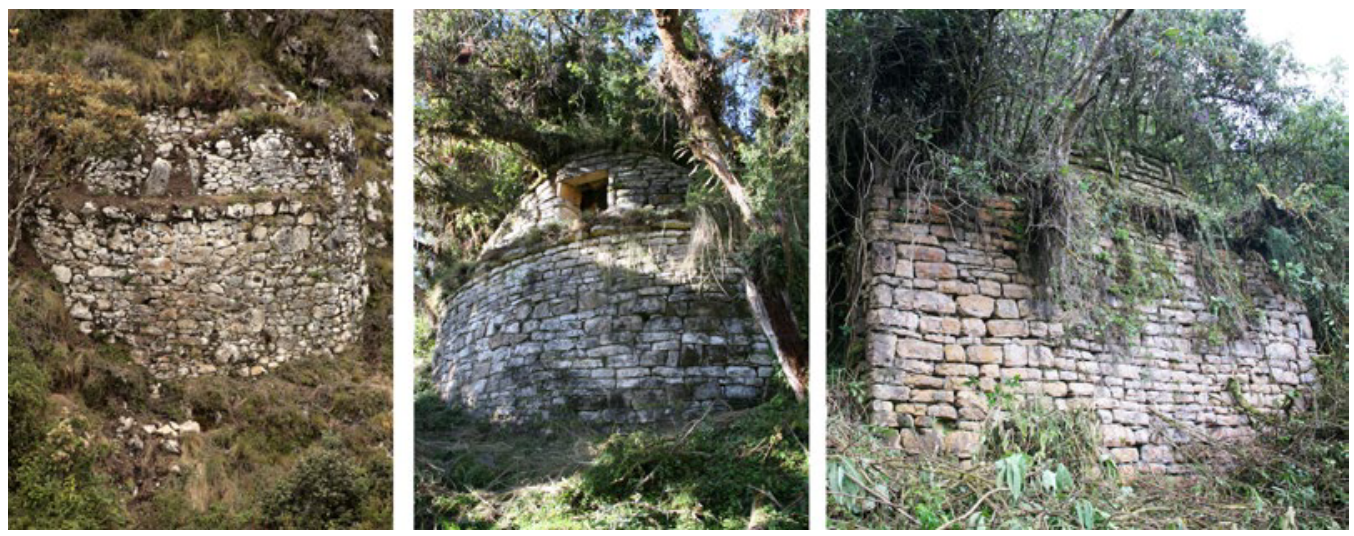

Figura 6. Bases plataformas. (izquierda) Estructura 61 de Monte Viudo; (centro) Estructura 199 de Monte Viudo; (derecha) base cuadrada de La Joya (foto por A. Guengerich).
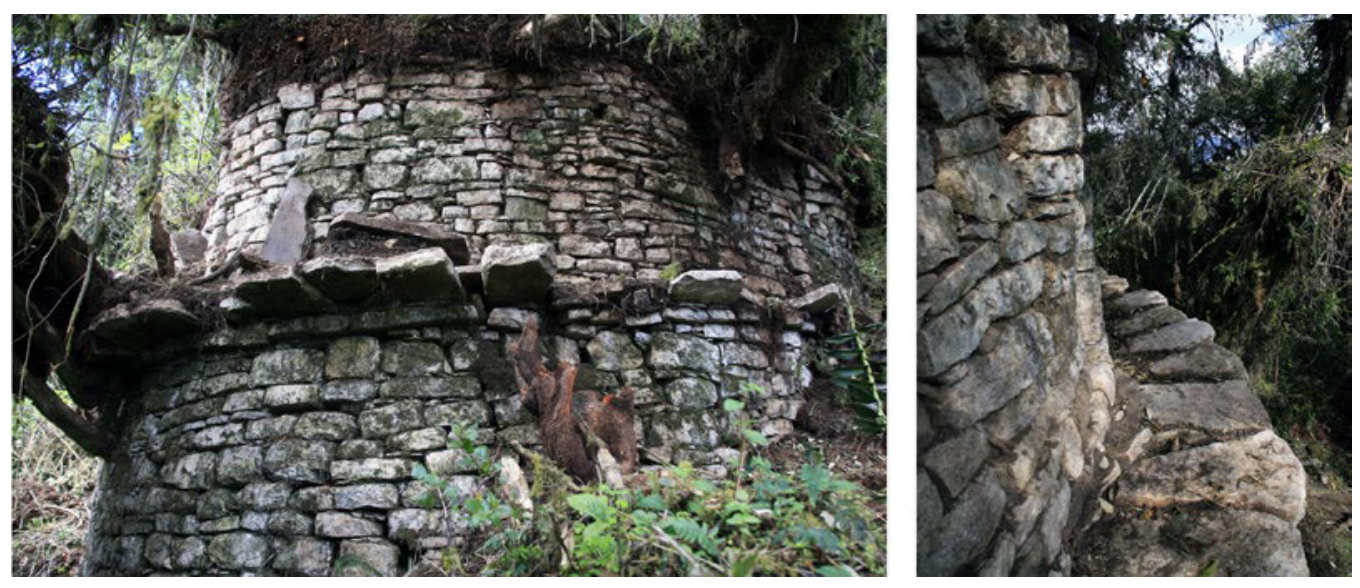

Figura 7. Cornisas de Monte Viudo: (izquierda) Estructura 200, (derecha) detalle de las lajas al frente de la portada, vistas desde arriba, Estructura 199 (foto por A. Guengerich).

daban a la entrada del edificio. Ya que las entradas siempre miran cuesta abajo, estas generalmente se ubican varios metros sobre la superficie del suelo; las cornisas, pues, representaban una manera de entrar el edificio desde la parte más alta de la cuesta. Además, algunos estudiosos han propuesto que las cornisas servían para alejar el agua de los cimientos y, de tal manera, proteger al edificio en el clima lluvioso de Chachapoyas (Bonavia 1968: 64-65). Finalmente, las cornisas probablemente tenían aspecto estético además de técnico. En Monte Viudo, por ejemplo, los edificios con mampostería fina contaban con cornisas de lajas talladas de aspecto delgado y ligeramente trapezoidal (Guengerich 2014a). Como explica Bonavia (1968: 65), la cornisa «daba a la estructura un sentido de horizontalidad, que dado la topografía irregular del terreno era muy difícil de lograr en las bases...y con esto se lograba un equilibrio visual interesante».

Pero el rasgo arquitectónico más conocido de Chachapoyas es, sin duda, el friso. Los frisos consistían en una franja con formas geométricas que rodeaban al edificio, normalmente limitándose a la mitad que miraba cuesta abajo. Podían ubicarse encima del dintel o en la base plataforma, pero se conservan pocos ejemplos en las paredes superiores debido a las condiciones de preservación que favorecen a las bases de los edificios. Los frisos también caracterizaban a otros tipos de construcciones del Período Intermedio Tardío, como las chullpas (v.g. von Hagen 2002b; Morales et al. 
2004) y las murallas de contención de andenes (v.g. Bonavia 1968; Koschmieder 2008). Existieron cinco motivos principales: los rombos, las grecas, el zigzag (Lerche 1995; Schjellerup 2005) y una forma espiralada (Ruíz Estrada 2013; Alexandrino y Guengerich 2017), además de un patrón de cuadrados en forma de «tablero de ajedrez» (Schjellerup 2005; Fabre 2006: 118) (Fig. 7). En algunos casos, en una misma franja se combinaron múltiples motivos (v.g. Ruiz Estrada 2013). Aunque los frisos más conocidos son los del Gran Pajatén, que lucen diseños de aves y de personas con tocados y manos entrelazadas (Bonavia 1968), en realidad son los únicos frisos zoomorfos o antropomorfos que se han encontrado en todo Chachapoyas. Es posible que estos reflejen los cambios asociados con la ocupación inka, época a la cual se remontan estos edificios (ibid.). De hecho, Schjellerup (2005) postula que los frisos florecieron en Chachapoyas durante esta época como expresión de identidad regional frente a los invasores. Pero también es posible que los frisos únicos del Gran Pajatén resultaran de tendencias estéticas o ideológicas autóctonas desarrolladas al nivel local.

\subsection{Campos reconocidos de la variación estilística arquitectónica}

La variación en los motivos de los frisos fue uno de los primeros aspectos de la cultura material donde se hizo evidente la gran variación geográfica que existió dentro de la extensa región conocida como Chachapoyas. En 1986, Lerche notó que los rombos se encontraban solo al norte de la ciudad actual de Leymebamba y las grecas al sur, mientras que los zigzags se extendían por toda Chachapoyas. Sobre la base de esta distribución, Schjellerup (2005: 448) postuló que Leymebamba - el único lugar en que se concentraron todos los motivos-, fue una zona de confluencia cultural que debe haber ejercido gran importancia política y/o étnica entre todas las sociedades locales de Chachapoyas. Aunque anteriormente se supuso que La Congona, al oeste de Leymebamba, era el único sitio que contaba con frisos con todos los motivos principales (Ruiz Estrada 1985), trabajos recientes en los sitios de Cerro Olán/San Pedro (Ruiz Estrada 2013) y el complejo de Tambillo (Guengerich 2014b: 140-141; Alexandrino y Guengerich 2017), indican que había varios lugares dentro de un radio de 15 kilómetros de Leymebamba, donde se emplearon todos los motivos a la vez. Es más, en ambos sitios también se ha registrado la presencia de un quinto tipo de friso, hasta entonces sin describir, que consistió en una forma espiralada y curvilínea (Ruiz Estrada 2013; Alexandrino y Guengerich 2017).

La falta de conocimiento del significado ideológico de los frisos para las sociedades de Chachapoyas dificulta la interpretación de estos patrones geográficos. Lerche (1995: 45-51) propuso que las formas de grecas, el zigzag y el rombo hacían referencia al cóndor, la serpiente y (el ojo de) el puma, los cuales, según Garcilaso (1966: 475), representaron las deidades principales de las poblaciones de Chachapoyas. Pero esta hipótesis es imposible comprobar mediante métodos arqueológicos y tampoco cuenta con afirmaciones de fuentes históricas más confiables.

A pesar de esta dificultad, la distribución de los frisos dentro de los sitios arqueológicos ofrece claves de interpretación acerca de su significado local. En todos los sitios descritos hasta el momento, los frisos solo caracterizan un porcentaje mínimo de edificios. En Monte Viudo, por ejemplo donde el buen estado de conservación posibilita una aproximación más fiel—, los frisos solo están presentes en 2,3\% de los trescientos edificios (Guengerich 2014a). Debido a que estos frisos se relacionan a los edificios más grandes, que están más cerca al centro del sitio y a los que cuentan con mampostería fina, se puede suponer que su uso estaba restringido a los grupos familiares de estatus social relativamente elevado ( $c f$. Ruiz Estrada 2013: 32). Su presencia, pues, en las fachadas debió haber señalado y reforzado la posición social de estas familias dentro de la comunidad. Es más, la presencia de los mismos motivos en edificios de propósito doméstico (v.g. EST199) y ceremonial (v.g. EST90) sugiere que tal vez los motivos particulares estuvieron ligados a algún aspecto de la identidad ritual. Se podría postular de manera inicial, que el empleo de los frisos estuvo ligado a las normas sociales de una comunidad local, mientras que el motivo del friso tuvo algún significado simbólico adicional, que estuvo ligado de alguna manera con divisiones sociogeográficas al nivel regional. 
La mampostería representa otro eje de variación geográfica de la arquitectura, pues variaba en cuanto a la presencia de la argamasa, el tamaño y la forma de las piedras, y especialmente al grosor de las lajas y su articulación (Fig. 8). Una de las variaciones más notables proviene del sitio de Chichita, en Luya, donde se encuentran varios edificios en los que las filas de piedra ascienden a manera de espiral, en vez de formar filas horizontales asentadas una sobre la otra (Koschmieder 2014: 81). Es probable que las variaciones en la mampostería resultaran de varios factores al nivel local y regional. A nivel del sitio, el estilo de la mampostería aparentemente variaba según el estatus social de los habitantes, el cual se evidencia en el trabajo invertido en el proceso de acarreo, esculpido y disposición de las piedras (Guengerich 2014b). Entre zonas geográficas, es posible que la variación en la mampostería además reflejara distintas identidades locales. Pero todavía son pocos los sitios que cuentan con fechas absolutas o relativas y es posible que cierta parte de la variación "geográfica» refleje, en realidad, cambios estilísticos a través del tiempo, como ha propuesto Schjellerup (2005: 370, 448).

Finalmente, la arquitectura doméstica también variaba en cuanto al tipo de piedra utilizada. Este es un tema que ha recibido menos atención. Es probable que la geología misma influenciara a la mampostería de distintas zonas geográficas, debido a las distintas propiedades físicas de piedra caliza, arenisca y pizarra. La mayoría de Chachapoyas yace sobre un estrato de roca calcárea, el cual proveyó las cavernas y los acantilados donde estas poblaciones enterraban sus difuntos. La piedra caliza, pues, predomina en la arquitectura de muchas zonas, entre ellas las cuencas del Utcubamba, del Atuén y del Sonche. Sin embargo, en las zonas donde abundan otros tipos de piedra, como la arenisca en Luya y los Chilchos (Lennon et al. 1989; Guillén 2000; Koschmieder 2012) (Fig. 8) y la pizarra en la cuenca del Abiseo (Bonavia 1968; Church 1997), estos materiales fueron seleccionados para elaborar la mayoría de construcciones. Aunque estas diferencias obviamente se originaron en el fondo en la distribución de los recursos naturales al nivel local, no se debe descartar la idea de que también tuvieran algún significado social o ideológico para sus arquitectos y habitantes, especialmente si se toma en cuenta la importancia de la piedra en el pensamiento de muchas sociedades andinas (v.g. Dean 2010).

\section{La diversidad local en las tradiciones arquitectónicas}

Aunque estos ejes de variación arquitectónica se han reconocido desde hace tiempo, curiosamente no han provocado ninguna crítica general a la idea de que toda Chachapoyas compartiera una sola tradición arquitectónica. Esta falta de autorreflexión se puede atribuir en gran parte al número limitado de estudios comparativos que se han realizado en la arqueología regional. Pero también resulta del enfoque predominante en el valle del Utcubamba antes del siglo XXI ( $c f$. Fabre et al. 2008). Afortunadamente, el aumento de nuevas publicaciones en base de estudios en zonas más alejadas, posibilita por primera vez las comparaciones sistemáticas de las diferencias locales en varios campos de cultura material, incluso la arquitectura. En este acápite reviso tres áreas socio-geográficas donde las investigaciones arqueológicas relativamente más desarrolladas, han permitido caracterizar ciertas tendencias que distinguían a las tradiciones locales de arquitectura doméstica: los distritos de Leymebamba-Chuquibamba, la provincia de Luya y la Cuenca del Sonche. Finalmente, considero las evidencias para la presencia de tradiciones arquitectónicas más allá de los territorios centrales de Chachapoyas.

\subsection{Los distritos de Leymebamba y Chuquibamba}

La zona donde se juntan las cuencas altas de los ríos Atuén y Chacahuayco — hoy en día compuesto por los distritos de Leymebamba y Chuquibamba ${ }^{3}$ - , es un terreno alto y accidentado. Consiste en su mayoría de kichwa y jalka, con elevaciones generalmente mayores a 2500 metros sobre el nivel del mar, pero mantiene zonas extensas de bosque montano en estado intacto. Se cuenta entre las áreas más estudiadas de Chachapoyas, debido a las obras extensas de Schjellerup (1992, 1997[2005]), así como de Keith Muscutt (1998; Muscutt et al. 1993), Marla Toyne (Epstein y Toyne 2016; Toyne y 

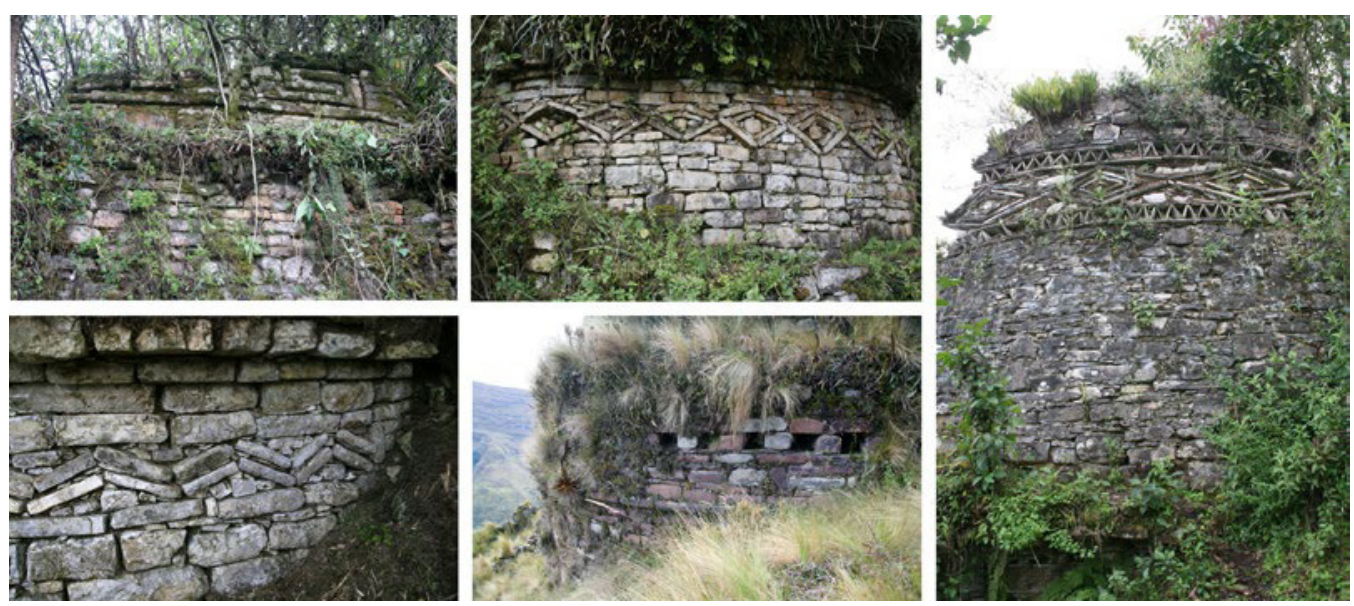

Figura 8. Variaciones en los estilos y motivos de frisos: (izquierda, arriba) greca, La Joya; (izquierda, abajo) zigzag, Monte Viudo; (centro, arriba) rombo; (centro, abajo) tabla de ajedrez, Vira Vira; (derecha) zigzag compuesto con rombo, Cerro Olán/San Pedro (foto por A. Guengerich).
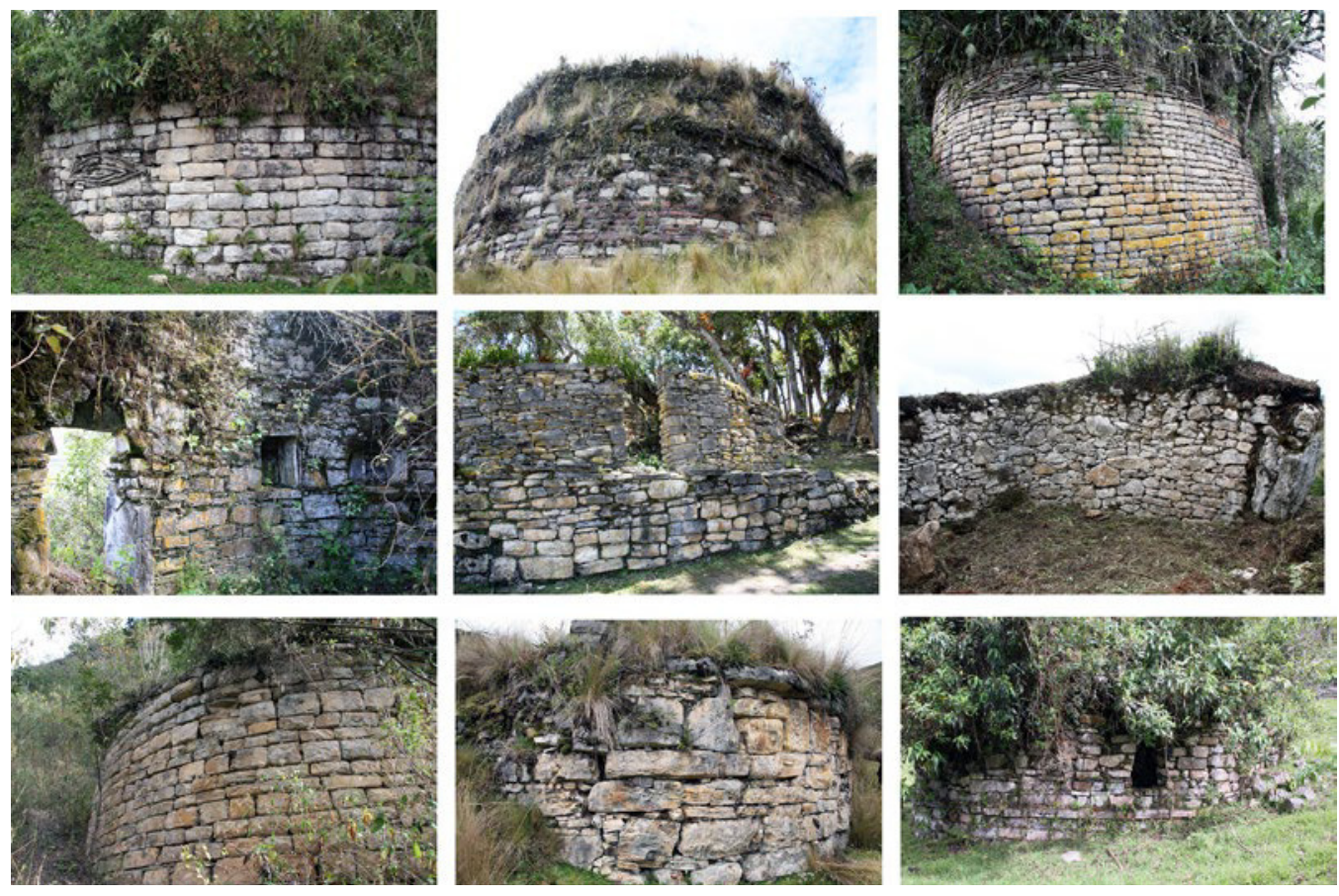

Figura 9. Variación en la mampostería: (de izquierda a derecha y de arriba a abajo) Yálape, Vira Vira, La Congona; Peña Calata, Kuélap, Monte Viudo; Macro, Bóveda, Cheto (foto por A. Guengerich).

Anzellini, este número), y la presente autora (Guengerich 2014a; Alexandrino y Guengerich 2017). Esta zona limita al sur con la cuenca del Chivane y el pueblo actual de Uchucmarca, un área que comparte ciertos aspectos de la arquitectura doméstica según los estudios de Donald Thompson (1976) y Abel Vega Ocampo (1982).

En esta zona abundaron los elementos de la «tríada arquitectónica chachapoya» — la base plataforma, la cornisa y el friso- - De hecho, la idea de que estos rasgos tipificaran a la arquitectura 
prehispánica de toda Chachapoyas, probablemente se debe en gran parte al número de estudios que se han realizado aquí, los cuales han dominado la literatura científica regional. Además, esta es la única parte de Chachapoyas en que se encuentran todos los motivos principales de frisos (Schjellerup 2005; Ruiz Estrada 2013; Alexandrino y Guengerich 2017), y hay pocos sitios que no cuentan con ninguna construcción con friso. Las bases plataforma también son frecuentes, y los sitios más grandes, como La Joya y Monte Viudo en el complejo de Tambillo, hasta dos tercios de las construcciones circulares cuentan con esta base (ibid.). Es más, estas llegan con frecuencia a alturas imponentes; por ejemplo, no son inusuales las bases de 3 metros o más de altura ( $v$.g. Alexandrino y Guengerich 2017).

Esta zona también contaba con rasgos arquitectónicos propios, que no se encuentran en otras partes de Chachapoyas. En muchos sitios, se ha registrado la presencia de cabezas clavas antropomorfas o zoomorfas (Vega Ocampo 1979; Muscutt et al. 1993; Muscutt 1998; Schjellerup 2005) (Fig. 9); sin embargo, casi todas han desaparecido debido al huaqueo. También se encuentran con frecuencia rampas de acceso que daban a la entrada de construcciones circulares (Muscutt 1998; Schjellerup 2005; Guengerich 2014b; pero ver también Guillén 2000 para un sitio de los Chilchos). Otro rasgo ampliamente difundido por Leymebamba-Chuquibamba, es una especie de cámara subterránea revestida con lajas o piedras (Thompson 1976; Vega 1979; Schjellerup 2005; Alexandrino y Guengerich 2017) (Fig. 10). Desafortunadamente, la falta de restos culturales dentro de estas cámaras dificulta averiguar cuál fue su propósito. En Kuélap —el único sitio donde se han registrado fuera de Leymebamba-Chuquibamba-, algunas estaban asociadas con restos humanos, lo cual refleja un propósito mortuorio, por lo menos en alguna etapa de su utilización (Narváez Vargas 2013). Por el contrario, Koschmieder (2014) propone que estas cámaras servían como almacenes de tubérculos en zonas de jalka como Chuquibamba, pues no se encuentran en Luya, que es una zona principalmente kichwa donde predominaba el cultivo del maíz.

Finalmente, la zona de Leymebamba-Chuquibamba también sobresale por la ausencia de entierros subterráneos, bajo los pisos de las viviendas, una práctica evidenciada en casi todas las otras partes de Chachapoyas (v.g. Ruiz Barcellos 2007; Koschmieder 2012; Crandall, este número). En los Andes y en otras sociedades pre-industriales, la práctica de "guardar» a los antepasados dentro de los espacios domésticos, reflejaba ideologías complejas asociadas con la importancia de la unidad familiar, la memoria social, la propiedad y las reglas de descendencia (Joyce y Gillespie 2000; Adams y King 2011). Esta diferencia, pues, entre esta y otras áreas de Chachapoyas, implica que las variaciones en la organización y la elaboración arquitectónica no solo representaron distintas tradiciones culturales o emblemas de identidad, sino también resultaron de diferencias ideológicas profundas sobre el significado del espacio doméstico y su relación con los habitantes.

\subsection{La provincia de Luya y Kuélap}

Los estudios de la provincia de Luya han sido mucho menos que los llevados a cabo en LeymebambaChuquibamba pero las investigaciones arqueológicas de Koschmieder $(2012,2014,2015)$ y las etnohistóricas de Ruiz Estrada (2011, este número), han afirmado que estas sociedades se distinguían en aspectos significativos. En varios sitios de Luya, la buena conservación permite vislumbrar detalles artesanales de la arquitectura local, como restos de enlucido e incluso pintura en las paredes interiores (Langlois 1940; Narváez Vargas 1996; Koschmieder 2012). También se han registrado mandíbulas de camélidos y astas de venado pegados en las paredes (ibid.). Aunque las astas se conocen de otras partes de Chachapoyas (v.g. von Hagen 2002a:142; Guengerich 2014b:143), las mandíbulas solo se han registrado en Luya. Sin embargo, según Koschmieder, el rasgo más característico de la arquitectura de Luya en la época preinka tardía fue una especie de peldaño semicircular ubicado al lado interior de la entrada (Fig. 11). El desnivel entre el piso interior y la superficie del terreno exterior tipificaba la arquitectura circular de toda Chachapoyas (Guillén 2000; Schjellerup 2005; Guengerich 2014b). El peldaño interior, pues, parece representar una técnica propia de 

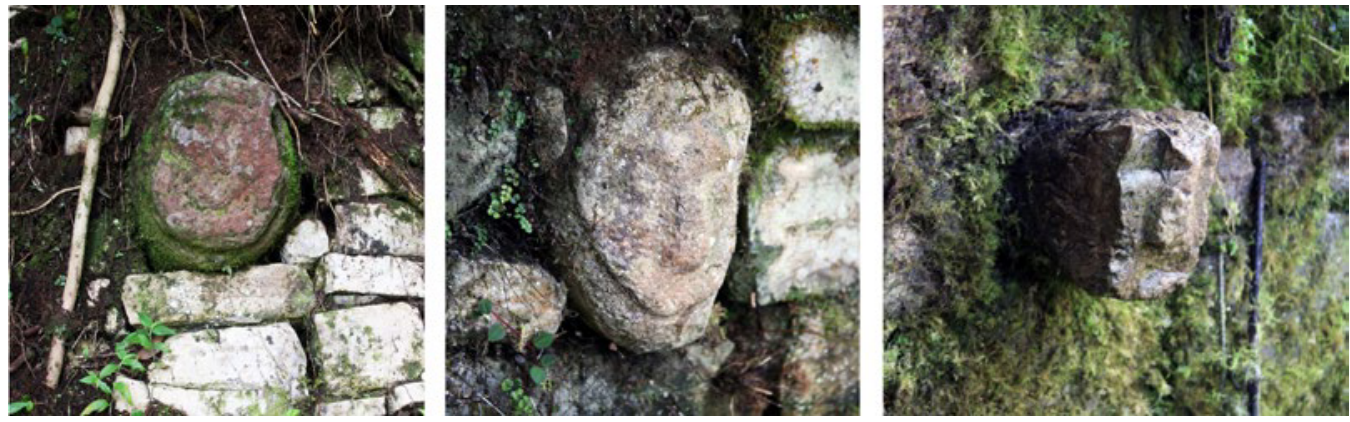

Figura 10. Cabezas clavas de la cuenca del Atuén: (izquierda) Molinete, (centro) La Vaquería, (derecha) La Joya (foto por A. Guengerich).

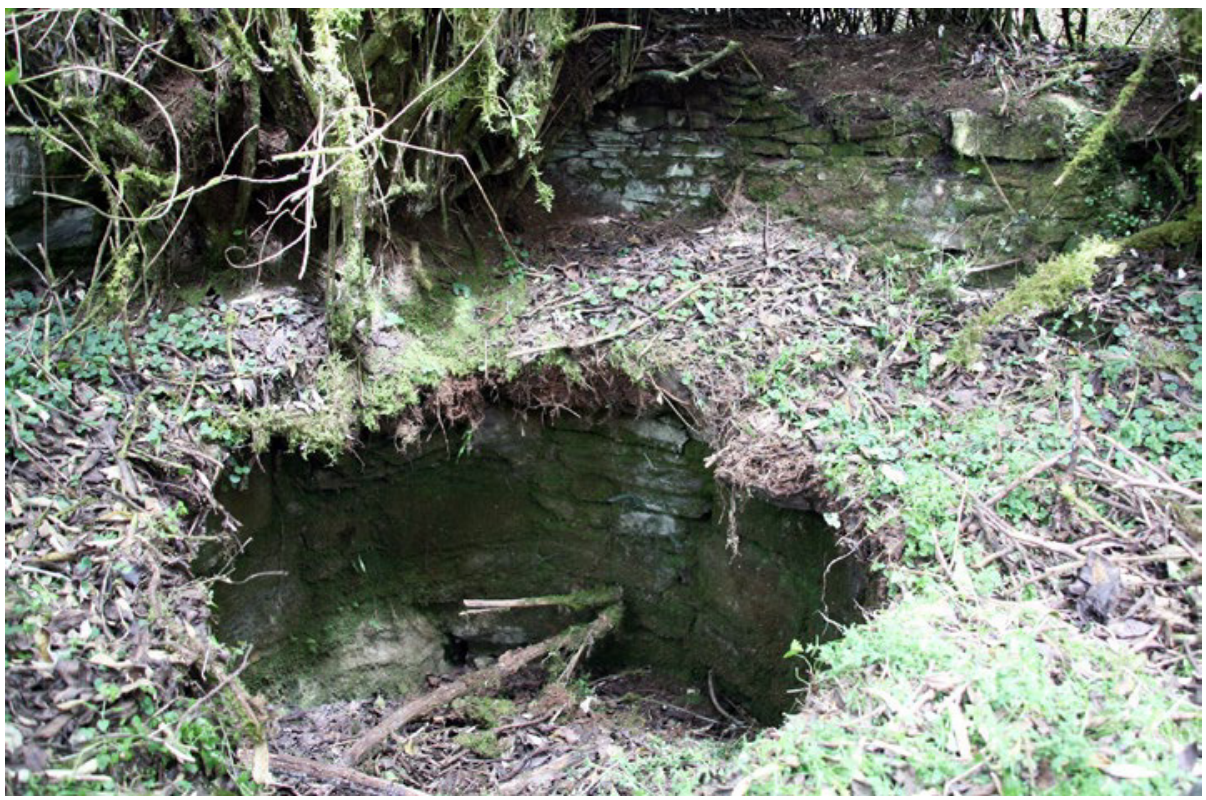

Figura 11. Cámara subterránea en el interior de una vivienda, Uchucmarca, cuenca del Pusac (foto por A. Guengerich).

Luya, mientras que en Leymebamba-Chuquibamba se utilizaban las escaleras (Schjellerup 2005), o lajas sobresalientes (Guengerich 2014a) al lado interior de las entradas.

La organización espacial de la arquitectura circular también refleja distintos patrones de actividades domésticas. Se encuentran batanes grandes en los interiores de muchas viviendas (Koschmieder 2012; Narváez Vargas 2013) y probablemente reflejan la importancia del maíz en la dieta local. En contraste, se los encuentran con poca frecuencia en Leymebamba-Chuquibamba, donde los tubérculos formaban la base de la dieta (Schjellerup 2005; Guengerich 2014a). Luya también se distingue por la ausencia de cualquier tipo de fogón, tushpa $a^{4} \mathrm{u}$ horno (Koschmieder 2012; Narváez Vargas 2013), los cuales se han encontrado en los interiores de las viviendas en muchos sitios de Leymebamba-Chuquibamba (Thompson 1976; Schjellerup 2005; Guengerich 2014a) (Fig. 12). Esto último hace suponer que muchas de las actividades domésticas entre las sociedades de Luya tomaban lugar al aire libre.

También hay algunas evidencias de que existió en Luya una forma distinta de techo, elaborado de piedra. La mayoría de los investigadores regionales actuales afirman el modelo de Davis 


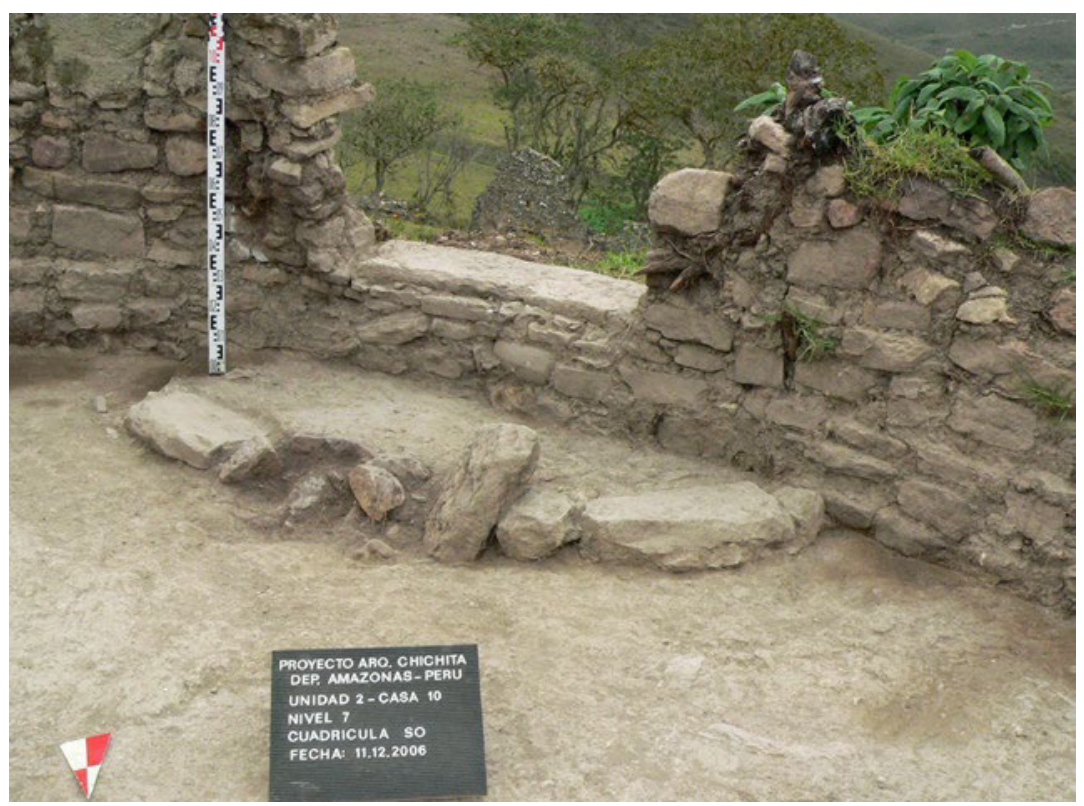

Figura 12. Interior de Casa 10 de Chichita, provincia de Luya, con detalle de peldaño semicircular (foto por K. Koschmieder).

(1996), quien plantea que los techos tenían forma cónica y consistían en una armadura de palos cubierta por paja, que se apoyaba directamente en los muros. Esta interpretación está apoyada por evidencias arqueológicas como las grandes cantidades de paja quemada que fueron encontradas al interior de muchas viviendas de Kuélap (Narváez Vargas 2013: 152) y la falta de huecos de poste encontrados en los interiores de los edificios circulares (Guengerich 2014b: 153; Koschmieder 2015: 27) . Sin embargo, algunos investigadores, como Louis Langlois (1940), han postulado que la forma ligeramente inclinada de las paredes superiores de muchas construcciones podría reflejar la presencia de un techo abovedado de piedra. Es más, el cronista Vásquez de Espinoza (1969: 283), notablemente, asevera la presencia de "casas...de hechura de media naranja» en la ciudad de Luya, un siglo después de la conquista española. Schjellerup (2005: 197) rechaza esta interpretación, aduciendo que esta evidencia fue el resultado del colapso gradual de las estructuras. No obstante, es posible que el techo abovedado fuera un rasgo propio solo de Luya y que la forma del techo representara otro eje de variación, que diferenciaba las tradiciones arquitectónicas en distintas partes de Chachapoyas.

Por otro lado, aunque Kuélap se ubica dentro del territorio de Luya, la arquitectura doméstica de este sitio cuenta con algunos rasgos que no son típicos de otros asentamientos de esta zona (Fig. 13). Los diámetros interiores de las viviendas generalmente variaban entre 7 y 9 metros (Narváez Vargas 1996: 101), más grande que en otros sitios de Luya u otras partes de Chachapoyas. También se distinguían por la presencia de un tipo de banqueta semicircular adosada a la pared trasera, la cual probablemente servía para dormir o descansar. Muchas construcciones también contaban con una especie de túnel cubierto sobre el nivel del piso, a los cuales Narváez Vargas (2013: 145) interpreta como cuyeros basándose en la presencia de los huesos encontrados adentro. Es difícil establecer si estos rasgos representen innovaciones locales propias de este sitio. Por un lado, hacen falta más estudios arquitectónicos en otras partes de Luya, más allá de Kuélap. Por otro lado, es posible que estos rasgos representen un desarrollo tardío, asociado con la presencia inka, pues la mayoría de los edificios circulares de este sitio fueron ocupados durante el Horizonte Tardío (Narváez Vargas 2013: 128). 


\subsection{La cuenca del río Sonche}

La zona más distintiva y la que origina más dudas acerca de la existencia de una «arquitectura chachapoya», es la cuenca del río Sonche. Esta zona cuenta con una historia de estudio breve, la cual data solo de los últimos diez años. Hasta ahora solo se han realizado investigaciones de los sitios de Chaquil, Fortaleza, Purun Llacta de Cheto y Purun Llaqta de Soloco, las cuales fueron dirigidas por Oliver Fabre (2006; Fabre et al. 2008), Arturo Ruiz Estrada (2004) y por James Crandall y sus colegas (Risco y Crandall, en prep.; Crandall, este número). Sin embargo, como explican Fabre et al. (2008: 275), el estudio de esta zona ofrece una oportunidad de ampliar nuestras ideas acerca de las formas «características» de cultura material de Chachapoyas en la época prehispánica pues, hasta la fecha, la mayoría de estudios se han enfocado en la margen izquierda del Utcubamba.

Muchos de los sitios en la cuenca del Sonche carecían de los elementos de la «tríada» clásica, y en casos donde sí se presenciaban, se manifestaban en formas que los diferenciaban de otras áreas de Chachapoyas. Por ejemplo, fueron pocas las construcciones que contaban con base plataforma, y donde sí existían, las bases eran de altura reducida, generalmente de menos de 1 metro (Fig. 14). En contraste con otras partes de Chachapoyas, las cornisas de la cuenca del Sonche eran angostas y frágiles. No podrían haber funcionado como pasarelas, sino que parecen haber cumplido una función más bien estética. Las cornisas no fueron necesarias para fines técnicos, pues las construcciones no se caracterizaban por bases altas que dificultaran el acceso (Fabre 2006:102). Es más, hasta ahora no se ha encontrado ni un friso en toda la cuenca del Sonche (ibid.: 120-121). Es posible que en algún momento sí existieran en las paredes supriores de algunas construcciones que no han sobrevivido (Fabre 2006: 120-121), pero la falta total de frisos incluso en las paredes inferiores donde normalmente se conservan hacer suponer que nunca estuvieron presentes. $\mathrm{Si}$, en otras partes de Chachapoyas, los motivos de los frisos comunicaban algún aspecto de la identidad social, se podría pensar que el rechazo intencional de este rasgo arquitectónico por los habitantes del Sonche representaría un intento de expresar una identidad local, que se diferenciara en aspectos fundamentales de las poblaciones vecindarios.

Tal vez el aspecto más notable de la arquitectura de esta zona es la cantidad de estructuras que no tenían forma circular — supuestamente el rasgo más característico de la arquitectura doméstica de Chachapoyas - . Entre ellas hay estructuras cuadrangulares, estructuras sub-rectangulares con esquinas redondeadas y estructuras que tienen la forma de «D» (Fabre 2006: 97-98; Fabre et al. 2008; Risco y Crandall, en prep. (fig. 11.2). Aunque estas formas son conocidas fuera de esta subregión, generalmente no se encuentra más de uno o dos ejemplares por sitio (v.g. Schjellerup 2005; Alexandrino y Guengerich 2017). En tales casos, algunos han planteado que esto reflejaría una influencia u origen inka (Langlois 1939; Thompson 1976; Guillén 2000: 4; Koschmieder 2008: 17), de todos modos, se puede suponer que fueron construcciones inusuales, que se originaron en circunstancias especiales o cumplieron funciones distintas (Alexandrino y Guengerich 2017). Por el contrario, en la cuenca del Sonche, las estructuras con formas no-circulares constituían un porcentaje notable de los sitios, lo cual sugiere que probablemente muchos representaran viviendas. En los sitios de Chaquil, Fortaleza y Soloco, por ejemplo, entre 0,4 y 5\% de las construcciones tenían forma rectangular o sub-rectangular, mientras que entre 6 y $10 \%$ tenían la forma de «D» (Fabre 2006: 97-98; Crandall, este número). La frecuencia de estas formas quiere decir que no solo la «tríada» sino también la característica más básica de la arquitectura de Chachapoyas —el plan circular-, no se puede considerar un elemento arquitectónico fundamental que uniera a todas las sociedades de esta región.

\subsection{Otras subregiones}

Más allá de estas tres áreas, aún se conocen muy poco las otras tradiciones locales de arquitectura doméstica de Chachapoyas. En la cuenca del río Abiseo, al extremo sur de la región, algunas diferencias en cuanto a los frisos indican la existencia de distintas normas culturales que gobernaban la arquitectura. No solo ocurren los únicos ejemplos de frisos figurativos en toda Chachapoyas, 


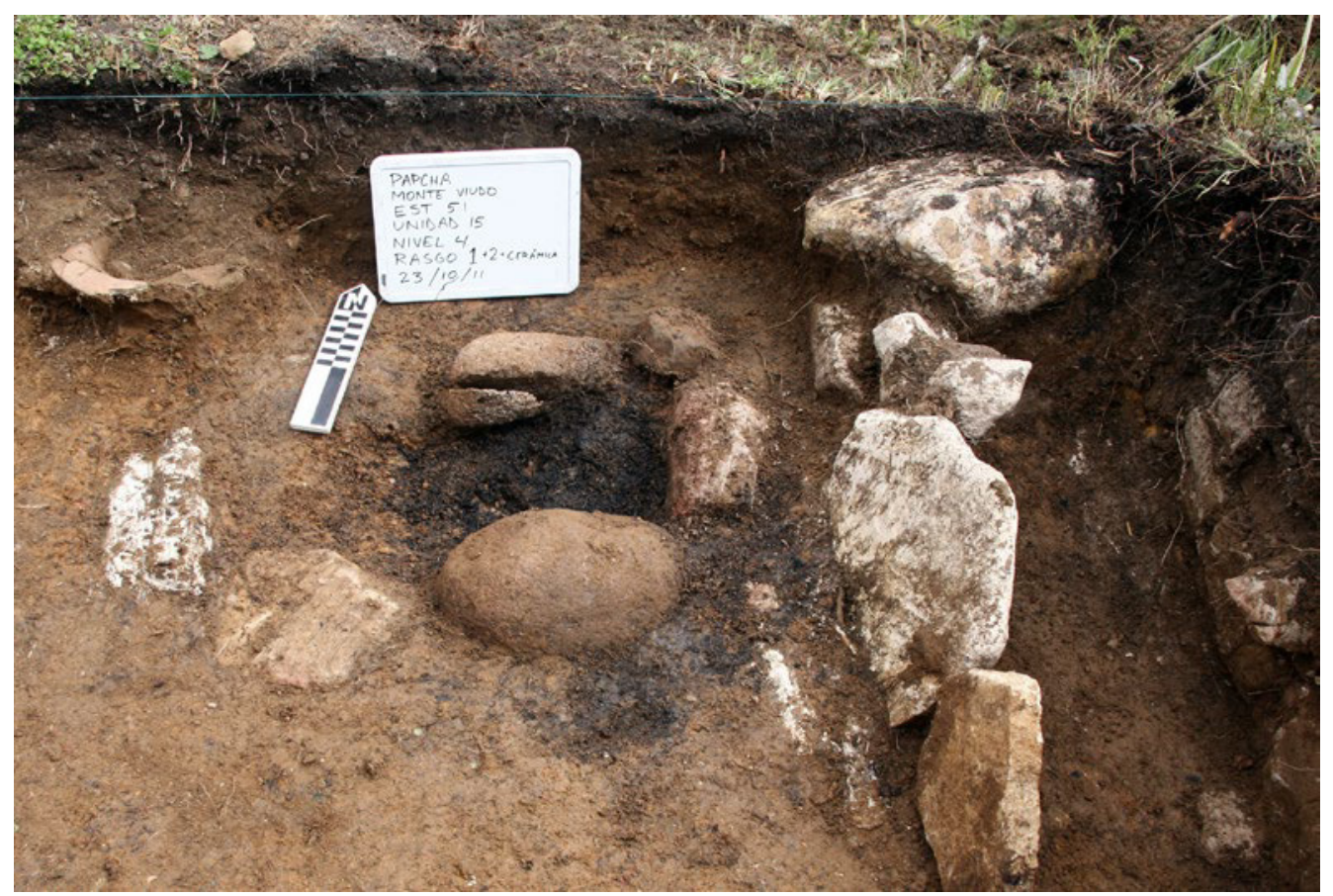

Figura 13. Una tushpa al interior de una casa (Estructura 51, Nivel 5, Monte Viudo) (foto por A. Guengerich).

Figura 14. Túnel cubierto y batán con chungo al interior de una vivienda en Kuélap (foto por A. Guengerich).

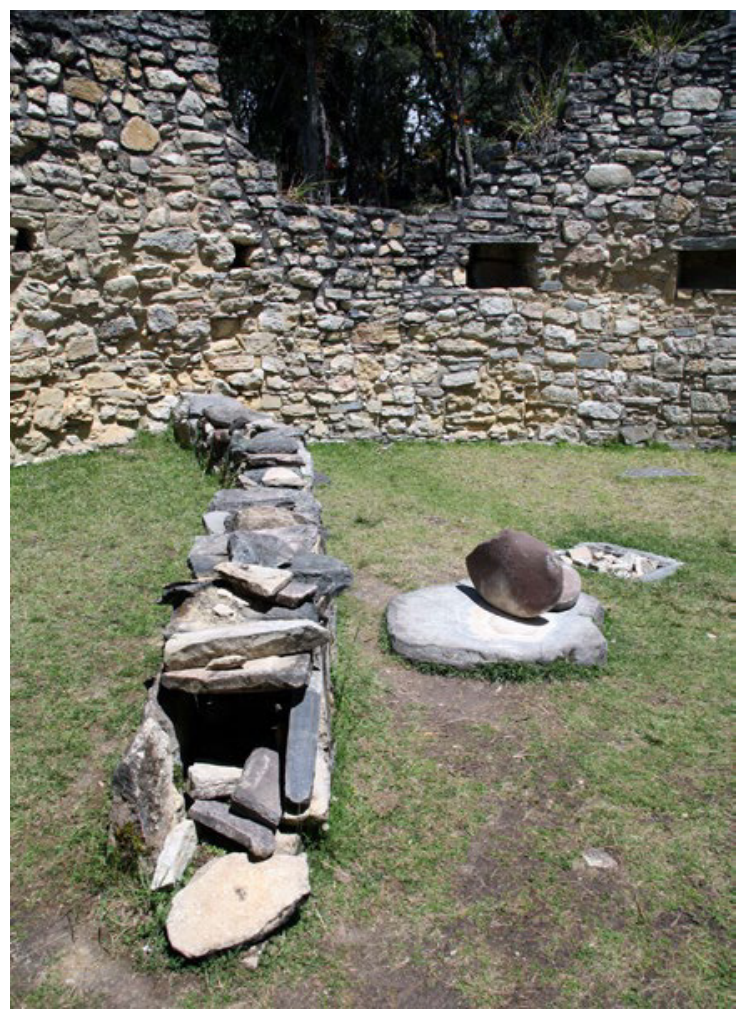


los cuales se encuentran en el Gran Pajatén (Bonavia 1968), sino que también se encuentran los únicos ejemplos de frisos que fueron elaborados al interior de las estructuras circulares (ibid. Church y Valle, este número Fig. 14). En la provincia de Rodríguez de Mendoza, las construcciones en sitios como Posic (Schjellerup et al. 2009: 23-54) y Wimba (McCray, este número) se caracterizan por muros bajos de menos de $1 \mathrm{~m}$ de altura, lo cual hace sospechar que la parte superior de las paredes fueron construidas de madera u otro material perecedero (Fig. 15), como la arquitectura vernácula de estas regiones de hoy en día. El sitio de Posic además, estaba asociado con plataformas circulares de 15 a 16 metros de diámetro? que carecían de muro superiores (Schjellerup et al. 2009), una forma arquitectónica que no se ha registrado en ninguna otra parte de Chachapoyas. Estas diferencias sugieren que las poblaciones de Rodríguez de Mendoza desarrollaron tradiciones arquitectónicas aún más alejadas de las tradiciones del Utcubamba y de las áreas del Sonche o el Abiseo.

Aunque se han llevado a cabo estudios en sitios habitacionales de Los Chilchos (Guillen 2000; Schjellerup et al. 2005), en la margen derecha superior del Utcubamba (Langlois 1939, 1940; Lerche 1986; Ruiz Estrada 2013) y en las cuencas de los ríos Huabayacu (Bueno y Cornejo 2009) y Abiseo (Bonavia 1968; Deza Rivasplata 1976; Church 1994, 1997), estos estudios no trataron la arquitectura doméstica como tema principal. En las zonas bajas al este y al norte de Chachapoyas, como Bagua y la montaña oriental de San Martín, tenemos escasa idea de las tradiciones arquitectónicas que caracterizaban al Período Intermedio Tardío. La ausencia de restos de piedra y el clima cálido de algunas de estas zonas plantea la posibilidad que se desarrollaran tradiciones de arquitectura perecedera, como las malocas documentadas en la época colonial entre los grupos cholones y jivitos de San Martín (Church 1996: 161-162).

\section{El significado social de la variación arquitectónica}

A pesar de estas evidencias, la idea de que la arquitectura doméstica habría formado uno de los elementos culturales compartidos por todas las sociedades de Chachapoyas persiste en los estudios regionales. En las descripciones de la arquitectura doméstica aún se emplean términos como «homogeneidad» (v.g. Schjellerup 2005: 64; Fabre et al. 2008: 273) y «uniformidad» (v.g. Lerche 1995: 29; Koschmieder y Gaither 2010: 6) para caracterizar los conjuntos arquitectónicos de sitios particulares, además de la arquitectura de la región en general. Ese no es el caso para otras formas de cultura material, por ejemplo, las prácticas funerarias, cuyas diferencias geográficas han sido reconocidas desde hace décadas.

Esta situación se debe en parte al estado incompleto de las fuentes de datos arqueológicas en Chachapoyas en el siglo XXI. Pero también resulta de tendencias intelectuales que están ampliamente difundidas en la arqueología en general. Debido a la larga historia disciplinaria de privilegiar a las construcciones monumentales en vez de las viviendas comunes y corrientes, se ha prestado poca atención a las variaciones que diferenciaban a las viviendas individuas o a las tradiciones arquitectónicas de comunidades distintas. Al contrario, los estudios de la arquitectura vernácula han sido dominados por la idea de que se puede interpretar como índice o reflejo de la identidad étnica (Aldenderfer y Stanish 1993; v.g. van Gijseghem y Vaughn 2008), una perspectiva que minimiza a las diferencias entre las construcciones a favor de su representación como una categoría con coherencia interna. Son pocos los estudios que examinan la arquitectura doméstica de una manera suficientemente detallada que permita reconstruir las circunstancias sociales y políticas que regían su creación (v.g. Bawden 1990; Bonnier 1997; van Gijseghem 2001; Henderson y Ostler 2005; Lau 2010). En los Andes, se encuentra la idea de una supuesta «homogeneidad» arquitectónica no solo en Chachapoyas, sino en muchas regiones de la sierra que carecían de tradiciones de arquitectura monumental durante el Período Intermedio Tardío (v.g. Bonavia 1978:401; Lavallée y Julien 1983: 66).

Sin embargo, estudios etnográficos de tradiciones vivas de arquitectura vernácula demuestran que muchas veces las diferencias que cargan gran significado para los miembros de estas sociedades, 


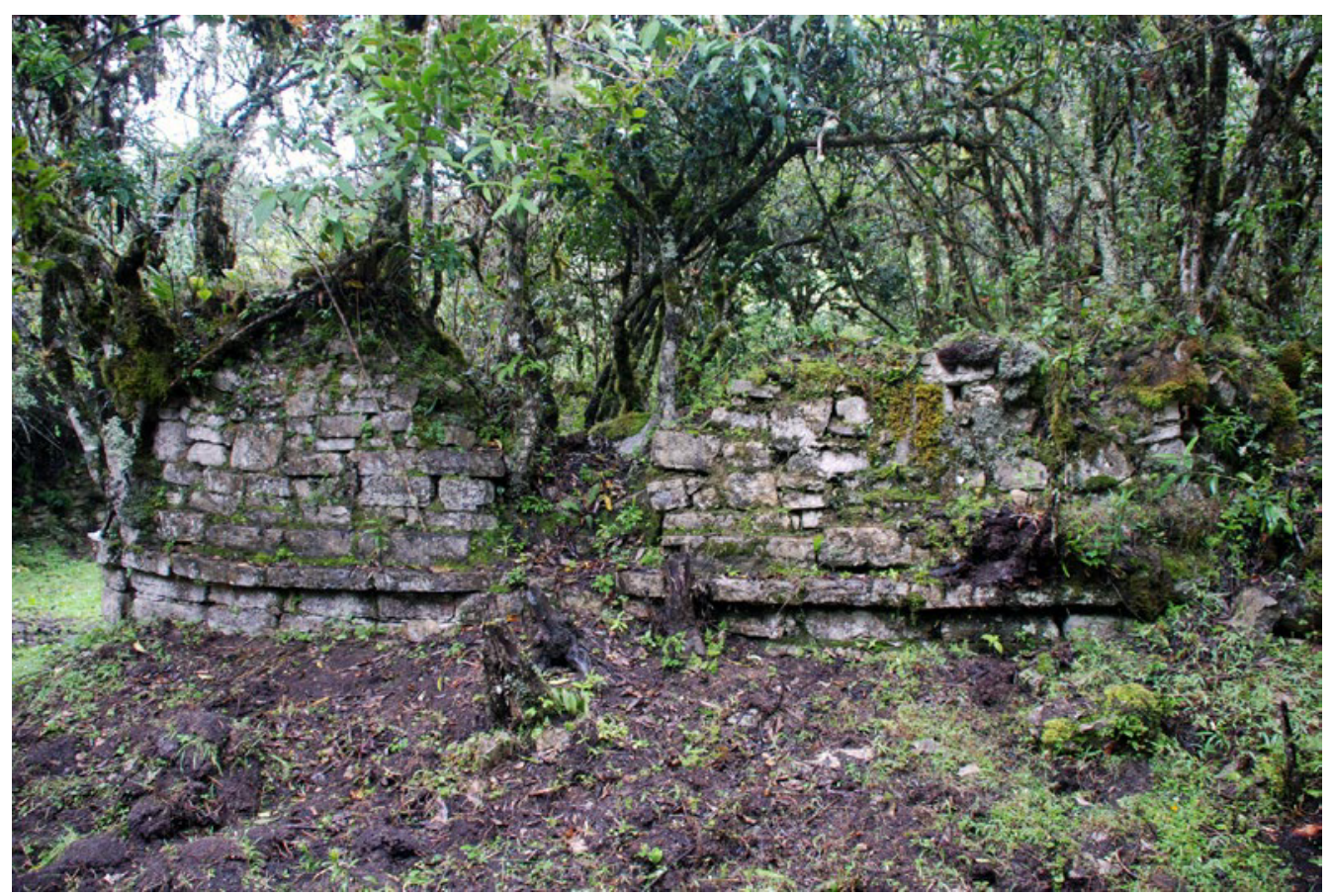

Figura 15. Estructura sub-rectangular del sitio de Gomal, valle de Sonche, con cornisa y base plataforma de reducido tamaño (foto por J. Crandall).

no siempre son fácilmente perceptibles por parte de individuos ajenos (Hubka 1979). En otras palabras, los detalles constructivos que pueden resultar «homogéneos» para los arqueólogos, eran frecuentemente legibles y significativos para los arquitectos y los habitantes de estas construcciones. Pero los detalles arquitectónicos registrados en sitios como Monte Viudo mediante una examinación minuciosa, demuestran que no es imposible percibir tales diferencias (Guengerich 2014a) (Fig. 16).

La gran importancia que tenía la arquitectura doméstica entre muchas poblaciones de Chachapoyas sugiere que estas diferencias habrían importado aún más en su contexto social original. Debido a la falta de construcciones monumentales en la mayoría de asentamientos (Guengerich 2015), las viviendas frecuentemente representaban la forma arquitectónica más impresionante en cada comunidad. De hecho, la arquitectura doméstica tenía aspectos realmente monumentales. La creación de cada construcción resultó de una inversión enorme de trabajo: según el modelo de Davis (1996), una construcción circular con diámetro interno de 5 metros requirió treinta toneladas de piedra caliza (sin contar la base) y veintiséis troncos de madera de 5 metros de largo, los cuales se acarreaban y cortaban sin la ayuda de herramientas de metal ${ }^{6}$. La construcción de un edificio tan grande seguramente involucraba la participación de muchos individuos y es probable que tomaba la forma de un evento parecido a la faena o el huasheo moderno, en que la familia que va a ocupar la vivienda provee la comida y la bebida para los que participan en sus construcciones (v.g. Brush 1977; Gose 1991; Malengreau 2009). La creación de cada vivienda, pues, debe haber sido un evento sobresaliente en la vida de las comunidades prehispánicas de Chachapoyas.

La importancia de la vivienda para muchas poblaciones de Chachapoyas en el Período Intermedio Tardío también se refleja en el alto nivel de detalle artesanal en detalles como los frisos, las hornacinas, las cabezas clavas, las astas de venado, los grabados geométricos y figurativos (Muscutt 1998; Schjellerup 2005) y la pintura. Muchos de estos detalles estaban presentes no solo en las construcciones asociadas a habitantes de estatus elevado, sino también en las construcciones 


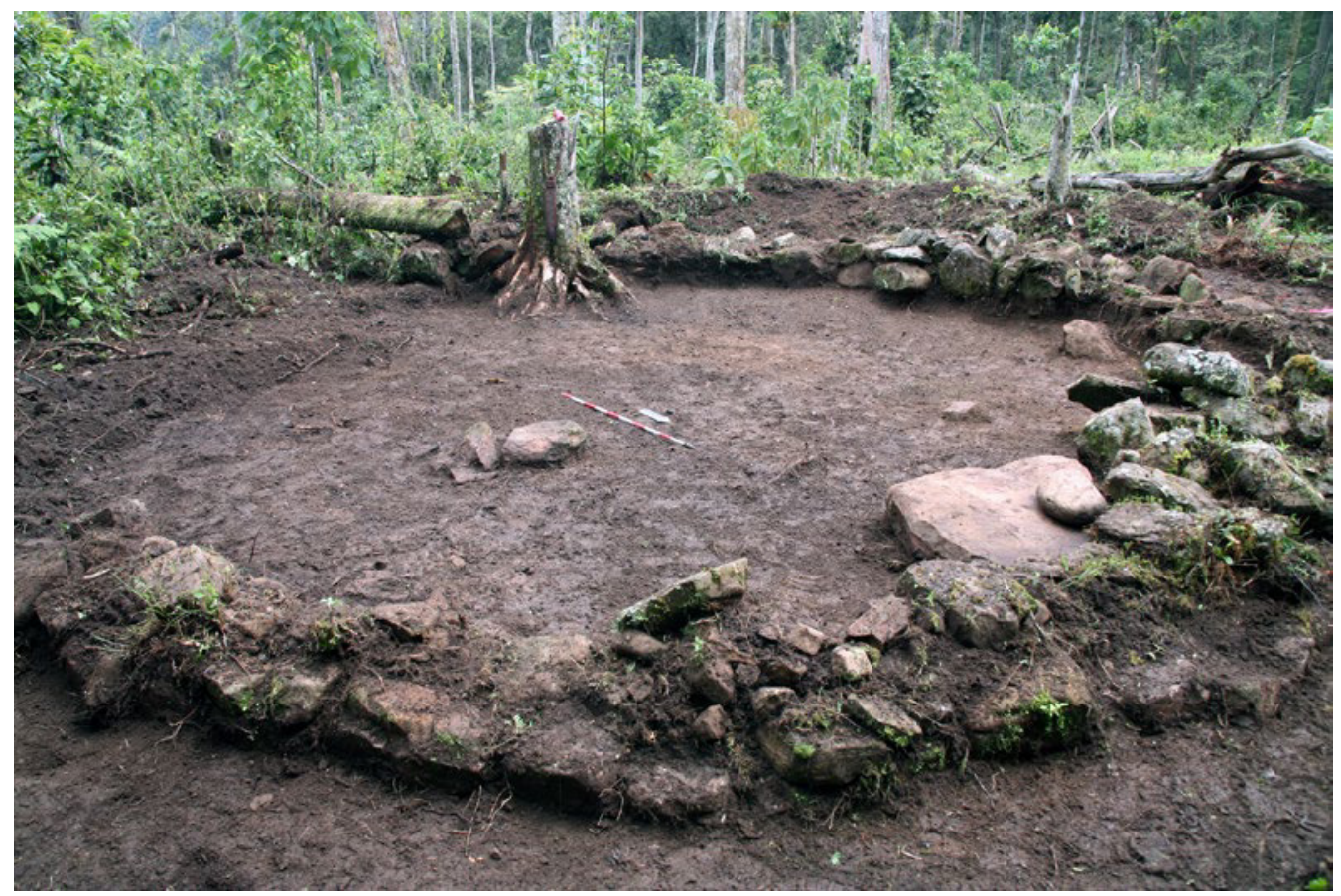

Figura 16. Paredes bajas de piedra en una vivienda en el Sector B de Posic (foto por A. Guengerich, reproducido con permiso de I. Schjellerup).

relativamente humildes encontradas en las zonas periféricas de los asentamientos, o caracterizadas por una calidad reducida de la mampostería (v.g. Guengerich 2014b). Parece, entonces, que la arquitectura doméstica no solo representó un lienzo en blanco en el que la élite podía comunicar su estatus social, sino también una institución cultural que tenía significado ideológico para todos los miembros de estas sociedades.

\section{Discusión: la arquitectura local frente a una «arquitectura chachapoya»}

Las representaciones de la arquitectura doméstica entre muchos de los estudios actuales tienen su origen en una época anterior en que aún era posible plantear la existencia de una sola «cultura chachapoya» compartida por toda la región. Sin embargo, como se ha demostrado en el presente artículo, una revisión de la literatura regional en el siglo XXI subraya la imposibilidad de mantener esta idea.

En primer lugar, los rasgos considerados más típicos de «la arquitectura chachapoya» - los frisos y las bases plataforma con cornisas-, no se extendían por toda Chachapoyas. Existieron diferencias notables en cuanto al estilo y a la función de estos tres rasgos, como las cornisas de propósito estético de la cuenca del Sonche, o los frisos interiores del Abiseo. En otros contextos, como la cuenca del Sonche, elementos como los frisos están completamente ausentes. En casos excepcionales, algunos de los rasgos del «tríada» se encuentran fuera de Chachapoyas, como los frisos zigzag registrados en varias chullpas del Alto Marañón de Huánuco y Áncash (Mantha y Malco 2004). Esta variabilidad significa que estos elementos no tienen su origen en ninguna identidad étnica compartida entre las supuestas sociedades chachapoyas, sino que cada grupo local se aprovechaba de algunos elementos — pero no todos_ que se manifestaban en la arquitectura de los grupos vecindarios. También es importante reconocer que no se ha registrado un solo sitio donde todos sus edificios estuvieron decorados con frisos, bases plataforma y cornisas; por el contrario, 
cada uno de estos rasgos solo están presentes en la minoría de las construcciones de un sitio. Es decir, el significado social de la arquitectura doméstica fue reconocido en primer lugar al nivel de la comunidad, donde reflejaba formas de identidad como la posición social o la filiación de linaje. La afiliación étnica o geográfica, pues, parece haber representado un nivel de identidad aparte, que se reflejaba en un conjunto de rasgos arquitectónicos.

A la vez, los únicos elementos arquitectónicos que sí se encuentran en todos los sitios habitacionales preinka de Chachapoyas — la forma circular y las construcciones de piedra—, también caracterizaban a la mayoría de tradiciones arquitectónicas regionales de la sierra andina contemporánea. Durante la segunda mitad del Período Intermedio Tardío, regiones tan diversas y tan alejadas como Junín (Bonnier 1997; DeMarrais 2001; Parsons et al. 2013), Tarma (Hastings 1985), Asto (Lavallée y Julien 1983), la cuenca del Titicaca (Arkush 2005; Frye y de la Vega 2005; Zovar 2012) y el altiplano de Chile (Nielsen 2002) desarrollaron tradiciones arquitectónicas basadas en el recinto circular de piedra. En realidad, antes de que se desarrollara la idea de que Chachapoyas se había distinguido por una identidad cultural particular (ver Church y Guengerich, este volumen), varios investigadores tempranos consideraron la arquitectura circular de esta región como una expresión de un patrón compartido entre muchas sociedades serranas (Bandelier 19840[1893]: 43; Reichlen y Reichlen 1950: 240-241), una observación que se ha perdido a través del tiempo.

Por supuesto, siempre se pueden encontrar excepciones a las categorías de cualquier sistema de organización, incluso la arqueología. No propongo, pues, que descartemos toda posibilidad de estudiar «la arquitectura de Chachapoyas» como una unidad analítica, especialmente al considerar las evidencias actuales de que estas poblaciones mantenían contacto frecuente entre sí. Sin embargo, es preciso reconocer que la arquitectura doméstica no se puede considerar un último refugio donde se pueda vislumbrar una unidad cultural compartida entre las sociedades preinka de Chachapoyas. La tendencia de descartar las diferencias reales que distinguían las tradiciones locales de arquitectura doméstica - en contraste con la práctica de reconocer a la variabilidad que existía en los aspectos como la cerámica y las prácticas funerarias-, ha permitido la continuación de una imagen falsa de homogeneidad de esta región. La deconstrucción de la idea de una sola «arquitectura chachapoya» ayudará a los esfuerzos actualmente en marcha, que pretenden exponer la gran diversidad cultural, ecológica y política que caracterizaba a Chachapoyas antes de la conquista de Tawantinsuyu.

\section{Conclusión}

El concepto aún vigente de la «arquitectura chachapoya» se debe en gran parte al retraso de los estudios regionales durante del siglo XX. Han sido pocos los estudios que se han enfocado en la arquitectura, especialmente en las áreas más allá del Utcubamba y hasta ahora no se ha realizado ninguna investigación sistemática que compare las tradiciones locales de distintas áreas. Estas tendencias han dificultado el registro de diferencias geográficas y han permitido que se consideren «típicos» los elementos que en realidad eran propios solo de localidades restringidas.

Todavía falta mucho que estudiar sobre la arquitectura doméstica de Chachapoyas, al igual que muchos aspectos de esta región extensa. Afortunadamente, muchas de estas cuestiones se pueden resolver a través de las investigaciones futuras y especialmente los esfuerzos de publicar, difundir y compartir los resultados. Debido a la buena conservación de la arquitectura doméstica en muchas zonas de esta región, Chachapoyas nos ofrece una oportunidad excelente de examinar las relaciones entre los grupos prehispánicos de los Andes y las tradiciones materiales y espaciales que desarrollaron.

\section{Agradecimientos}

Quisiera agradecer a los miembros del Boletín de Arqueología PUCP y los revisores anónimos por sus comentarios y sugerencias en las versiones anteriores de este trabajo. Este artículo fue producto de muchos viajes con compañeros quienes me han introducido a una multitud de sitios arqueoló- 
gicos. Agradezco en particular a Eyrin Chávez Chancahuana y Rob Dover, además de Lenis Valqui, Manuel Malaver, Karyna Reyes, Keith Muscutt y Adriana von Hagen. También agradezco a James Crandall y Klaus Koschmieder por compartir sus fotos, y a todos los miembros de los equipos de PAPCHA y PATA quienes han participado en los trabajos en Tambillo.

\section{Notas}

${ }^{1}$ Utilizo el término «Chachapoyas» de acuerdo con el sentido planteado en el capítulo de conclusión de este volumen, para hacer referencia a la zona geográfica abarcada por la provincia inka, según los cronistas espańoles y, después, Rowe (1946).

${ }^{2}$ Utilizo el término «regional» para hacer referencia a la extensa zona arqueológica de Chachapoyas (ver Nota 1) y el término «local» para hacer referencia a las subregiones o áreas más limitadas con cierta integridad geográfica o arqueológica, como por ejemplo los Chilchos, Luya, Abiseo, etc.

${ }^{3}$ Los distritos (y pueblos) de Chuquibamba y Leymebamba han mantenido una estrecha relación social y política desde por lo menos la época colonial y tal vez inka, o antes (Schjellerup 2005) - relación que se manifiesta hoy en día en varios líos sin resolver sobre territorios intermedios-. Aunque esta zona pertenece a dos zonas orográficas (las cuencas del Atuén y el Chacahuayco), la concentración de sitios prehispánicos sugiere que las poblaciones interactuaban frecuentemente entre sí, igual en el pasado, como hoy.

${ }^{4} \mathrm{La}$ tushpa es una forma tradicional de fogón que se evidenciaba en Amazonas con frecuencia hasta el siglo XX. Consta de unas pocas rocas grandes que forman un círculo; al interior se coloca material combustible como paja o leña y se coloca la olla encima.

${ }^{5}$ Los únicos ejemplos de posibles huecos de poste en los interiores de estructuras circulares están asociados a sitios ocupados durante la época inka (Moyambol [Schjellerup 2005: 230] e Inticancha [Thompson 1976]), aunque han sido pocos los estudios regionales donde se ha registrado esta información. La falta de evidencias de contextos anteriores al Horizonte Tardío es especialmente notable en vista de la importancia cosmológica de postes centrales en otras sociedades de los Andes septentrionales (Caillavet 2000: 142; Henderson y Ostler 2005).

${ }^{6}$ Esta cifra se basa en paredes de 0,5 metros de espesor y 2 metros de altura. Se utiliza la gravedad específica promedio de piedra caliza $\left(2,5 \mathrm{~g} / \mathrm{cm}^{2}\right)$. Unos 5000 kilos han sido sustraídos de la cifra total debido al espacio vacío que se encuentra entre las piedras de la mampostería. En la reconstrucción de Davis, las maderas están atadas a las paredes a cada 0,5 metros y los lados del techo mantienen un ángulo de 60 grados.

\section{REFERENCIAS}

Adams, R. y S. King

2011 Residential burial in global perspective, en: R. Adams y S. King (eds.), Residential burial: a multiregional exploration, Archaeological Papers of the American Anthropological Association, 1-16, Washington, D.C.

Aldenderfer, M. y C. Stanish (eds.)

1993 Domestic architecture and complementarity in the south central Andes, University of Iowa, Iowa City.

Alexandrino, G. y A. Guengerich

2017 Informe de los resultados del Proyecto Arqueológico Tambillo, Temporada 2016., informe entregado al Ministerio de Cultura del Perú.

Arkush, E.

2005 Colla fortified sites: warfare and regional power in the Late Prehispanic Titicaca basin, Peru, tesis de doctorado, Departamento de Antropología, Universidad de California, Los Angeles. 


\section{Bandelier, A.}

1940 Los indios y las ruinas aborígenes de Chachapoyas en el norte del Perú, Chaski 1(2),13-49.

[1893]

Bawden, G.

1990 Domestic space and social structure in pre-Columbian northern Peru, en: S. Kent (ed.), Domestic architecture and the use of space: a cross-cultural study, 153-171, Cambridge University Press, Cambridge.

\section{Bonavia, D.}

1968 Las ruinas del Abiseo, Universidad Peruana de Ciencias y Tecnología, Lima.

1978 Ecological factors affecting the urban transformation in the last centuries of the pre-columbian era, en: D. Browman (ed.), Advances in Andean Archaeology, 393-411, The Hauge, Mouton.

Bonnier, E.

1997 Morfología del espacio aldeano y su expresión cultural en los Andes Centrales, en: E. Bonnier y H. Bischof (eds.), Archaeologica Peruana 2: arquitectura y civilización en los Andes prehispánicos, 28-41, Sociedad Arqueológica Peruano-Alemana, Reiss-Museum, Mannheim, Germany.

Brush, S.

1977 Mountain, field and family: the economy and human ecology of an Andean valley, University of Pennsylvania Press, Philadelphia.

Bueno, A. y M. Cornejo García

2009 Arqueología de la cuenca del río Guabayacu. Región San Martin, Perú, Investigaciones Sociales 13(23), $15-58$.

Church, W.

1994 Early occupations at Gran Pajatén, Peru, Andean Past 4, 281-318.

1996 Prehistoric cultural development and interregional interaction in the tropical Montane Forests of Peru, tesis doctoral, Department of Anthropology, Yale University.

1997 Más allá del Gran Pajatén: conservando el paisaje prehispánico Pataz-Abiseo, Revista del Museo de Antropología, Arqueología e Historia 7, 205-248 (e.p.)

\section{Church, W. y A. von Hagen}

2008 Chachapoyas: cultural development at an Andean cloud forest crossroads, en H. Silverman y W. Isbell (eds.), Handbook of South American Archaeology, 903-926, Springer, New York.

Crandall, J.

2012 Chachapoya eschatology: spaces of death in the northern Andes, Baessler-Archiv Band 60(2012), 1-17.

Davis, M.

1996 La casa redonda y Yurac-Urco, Auto-publicado, Noelville, Ontario.

Dean, C.

2010 A culture of stone: Inka perspectives on rock, Duke University, Durham.

DeMarrais, E.

2001 The architecture and organization of Xauxa settlements, en T. D’Altroy y C. Hastorf (eds.), Empire and domestic economy, 115-153, Kluwer Academic/Plenum, New York.

Deza Rivasplata, J.

1976 La Playa: un complejo arqueologico en la cuenca del Abiseo, Boletin del Seminario de Arqueologia 17-18, 43-50.

Epstein, Lori y J. M. Toyne

2016 When space is limited: a spatial exploration of preh-hispanic Chachapoya mortuary and ritual microlandscape, en: A. J. Osterholtz (ed.), Theoretical Approaches to Analysis and Interpretation of Commingled Human Remains, 97-124, Springer, New York.

Fabre, $\mathrm{O}$.

2006 Contribution à l'archéologie de la région Chachapoya, Pérou, tésis de doctorado, Université ParisSorbonne.

Fabre, O., J. Loup Guyot, R. Salas Gismondi, M. Malaver Pizarro y E. Maniero

2008 Los chachapoya de la región de Soloco: Chaquil, del sitio de hábitat a la cueva funeraria, Bulletin de l'Institut Français d'Études Andines 37(2), 271-292. 
Frye, K. y E. de la Vega

2005 The Altiplano period in the Titicaca Basin, en: C. Stanish, A. Cohen y M. Aldenderfer (eds.), Advances in Titicaca Basin Archaeology I, 173-184, Cotsen Institute of Archaeology, Universidad de California, Los Angeles.

Garcilaso de la Vega, Inca

1966 Royal commentaries of the Incas, and general history of Peru [traducido por H.V. Livermore], University of [1609] Texas, Austin.

Gose, P.

1991 House rethatching in an Andean annual cycle: practice, meaning, and contradiction, American Ethnologist 18(1), 39-66.

\section{Guengerich, A.}

2014a The architect's signature: the social production of a residential landscape at Monte Viudo, Chachapoyas, Peru, Journal of Anthropological Archaeology 34, 1-16.

2014b Monte Viudo: residential architecture and the everyday production of space in a Chachapoya community, tesis de doctorado, Departamento de Antropología, Universidad de Chicago.

2015 Settlement organization and architecture in Late Intermediate Period Chachapoyas, Northeastern Peru. Latin American Antiquity 26(3), 362-381.

Guillén, $S$.

2000 Informe final del proyecto evaluación y delimitación del sitio arqueológico Llaqtacocha, entregado al Instituto Nacional de Cultura, Lima.

von Hagen, A.

2002a Pueblo de las nubes, en: E. González y R. León (eds.), Chachapoyas: el reino perdido, 24-261, AFP Íntegra, Lima.

2002b Chachapoya iconography and society at Laguna de los Cóndores, Peru, en: H. Silverman y W. Isbell (eds.), Andean Archaeology, Volume II: art, landscape, and society, 137-155, Kluwer Academic/Plenum, New York.

Hastings, C.

1985 The eastern frontier: settlement and subsistence in the Andean margins of Central Peru, Vol.1, tesis de doctorado, Departamento de Antropología, Universidad de Michigan.

\section{Henderson, H. y N.Ostler}

2005 Muisca settlement organization and chiefly authority at Suta, Valle de Leyva, Colombia: a critical appraisal of native concepts of house for studies of complex societies, Journal of Anthropological Archaeology 24, 148-178.

Horkheimer, $\mathrm{H}$.

1959 Algunas consideraciones acerca de la arqueología en el valle del Utcubamba, Actas del II Congreso Nacional de Historia del Perú, 71-90.

Hubka, T.

1979 Just folks designing: vernacular designers and the generation of form, Journal of $\mid$ Architectural Education 32(3), 27-29.

Joyce, R. y S. Gillespie (eds.)

2000 Beyond kinship: social and material reproduction in house societies, University of Pennsylvania, Philadelphia.

Kauffman, F. y G. Ligabue

2003 Los chachapoya(s): moradores ancestrales de los Andes amazónicos peruanos, Universidad Alas Peruanas, Lima.

Koschmieder, K.

2008 Proyecto arqueológico Jucusbamba: primera temporada, informe entregado al Instituto Nacional de Cultura, Lima.

2012 Jucusbamba: investigaciones arqueológicas y motivos Chachapoya en el norte de la Provincia de Luya, Departamento Amazonas, Perú, Auto-publicado, Lima.

2014 Los orígenes y el desarrollo de la organización socio-política de la cultura chachapoya: una mirada desde la provincia de Luya, Departamento Amazonas, Perú, en: S. Rostain (ed.), Antes de Orellana-Actas de

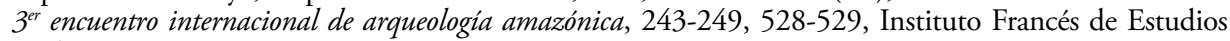
Andinos, Quito. 
2015 Asentamientos chachapoyas en el norte de la provincia de Luya, departamento de Amazonas, Arqueología y Sociedad 28, 71-114.

Koschmieder, K. y C. Gaither

2010 Tumbas de guerreros chachapoya en abrigos rocosos de la provincia de Luya, departamento de Amazonas, Arqueología y Sociedad 22, 9-38.

Langlois, L.

1939 Utcubamba: investigaciones arqueológicas en este valle del departamento de Amazonas, Revista del Museo Nacional 8(2), 224-249.

1940 Utcubamba: investigaciones arqueológicas en este valle del departamento de Amazonas, Revista del Museo Nacional 9(1), 34-71.

Lau, G.

2010 House forms and Recuay culture: residential compounds at Yayno (Ancash, Peru), a fortified hilltop town, AD 400-800, Journal of Anthropological Archaeology 29, 327-351.

Lavallée, D. y M. Julien

1983 Asto: curacazgo prehispánico de los Andes Centrales, Instituto de Estudios Peruanos, Lima.

Lennon, T., W.B. Church y M. Cornejo

1989 Investigaciones arqueológicas en el parque nacional Río Abiseo. Editorial Los Pinos 1962:43-56.

Lerche, P.

1986 Häuptlingstum Jalca: bevölkerung und ressourcen bei der vorspanischen Chachapoya, Peru, Dietrich Reimer, Berlin.

1995 Los Chachapoya y los símbolos de su historia, Ediciones y Servicios Gráficos César Gayoso, Lima.

Malengreau, J.

2009 Parientes, paisanos y ciudadanos en los Andes de Chachapoyas, Instituto Francés de Estudios Andinos, Lima.

Mantha, A.y H. Malco

2004 El sitio de Rapayán y su diversidad arquitectónico durante los períodos tardíos de la prehistoria andina, en: B. Ibarra (ed.), Arqueología de la sierra de Ancash: perspectivas y propuestas, 1-22, Instituto Cultural Runa, Lima.

Morales, R., L. Valle Álvarez y W. Church

2004 Arquitectura chachapoya: análisis de la tecnología constructiva e iconográfica, Sian 9(15), 16-17.

Muscutt, K.

1998 Warriors of the clouds: a lost civilization in the upper Amazon of Peru, University of New Mexico, Albuquerque.

Muscutt, K., V. Lee y D. Sharon

1993 Vira Vira: a «new» Chachapoya site, Sixpac Manco publications, Wilson, WY.

Narváez Vargas, A.

1996 La fortaleza de Kuélap 1, Arkinka 12, 92-108.

2013 Kuélap: centro del poder politico religioso de los Chachapoyas, en: F. Kauffman (ed.), Los Chachapoyas, 87-159, Banco de Crédito, Lima.

Nielsen, A.

2002 Asentamientos, conflicto y cambio social en el altiplano de Lípez (Potosí), Revista Española de Antropología Americana 32, 179-205.

Parsons, J., C. Hastings y R. Matos Mendieta

2013 Prehispanic settlement patterns in the upper Mantaro, Junin, Peru, volume 2: the Wanka Region, Museum of Anthropology, University of Michigan, Ann Arbor.

Reichlen, H. y P. Reichlen

1950 Recherches archéologiques dans les Andes du haut Utcubamba, Journal de la Société des Américanistes 39, 219-246.

Risco, L. y J. Crandall

sf. Informe de investigaciones en Purum Llacta de Soloco, informe entregado al Ministerio de Cultura, Lima, en preparación. 
Rowe, J.

1946 Inca Culture at the Time of the Spanish Conquest. En J. Steward (ed.), Handbook of South American Indians, Volume 2: the Andean Civilizations, 183-330, Smithsonian Institution, Washington, D.C.

Ruíz Barcellos, J.

2007 Arqueología y arquitectura de Yálape en la provincia de Amazonas, Arkinka 136, 84-91.

Ruíz Estrada, A.

1985 Los monumentos arqueológicos de Leymebamba, Boletín de Lima 7(42), 69-82.

2004 Purumllacta: un centro administrativo incaico en Chachapoyas, Investigaciones Sociales 8(13), 73-84.

2011 Amazonas: arqueología e historia, Universidad Alas Peruanas, Lima.

2013 Investigaciones arqueológicas en San Pedro, Chachapoyas-Peru, Investigaciones Sociales 17(31), 27-38.

Schjellerup, I.

1992 Patrones de asentamiento en las faldas orientales de los Andes de la región de Chachapoyas, en: D. Bonavia (ed.), Estudios de arqueologia peruana, 355-373, FOMCIENCIAS, Lima.

1997 Incas and Spaniards in the conquest of the Chachapoyas: archaeological and ethnological research in the northeast Andes of Peru, Göteburg University/National Museum of Denmark, Göteburg.

2005 Incas y españoles en la conquista de los Chachapoya, Pontificia Universidad Católica de Perú, Lima.

Schjellerup, I., M. Kamp Sørenson, C. Espinoza, V. Quipuscoa y V. Peńa

2003 Los valles olvidados: pasado y presente en la utilización de recursos en la ceja de selva, Perú, Ethnographic Monographs, no.1, National Museum of Denmark, Copenhagen.

Schjellerup, I., V. Quipuscoa, C. Espinoza, V. Peña y M. Kamp Sørenson

2005 Redescubriendo el valle de los Chilchos: condiciones de vida en la ceja de selva, Perú, Ethnographic Monographs, no.2, National Museum of Denmark, Copenhagen.

Schjellerup, I., C. Espinoza, J. Rollefson, V. Quipuscoa, M. Kamp Sørenson yV. Peńa

2009 La ceja de montaña: un paisaje que va despareciendo, Ethnographic Monographs, no.3. National Museum of Denmark, Copenhagen.

Thompson, D.

1976 Prehistory of the Uchucmarca Valley in the north highlands of Peru, Proceedings of the 41st international Congress of Americanists 99-106, 99-130.

van Gijseghem, $\mathrm{H}$.

2001 Household and family at Moche, Peru: an analysis of building and residence patterns in a prehispanic urban center, Latin American Antiquity 12(3), 257-273.

van Gijseghem, H. y K.Vaughn

2008 Regional interaction and the built environment in middle-range societies: Paracas and early Nasca houses and communities, Journal of Anthropological Archaeology 27, 111-130.

Vásquez de Espinoza, A.

1969 Compendio y descripción de las Indias Occidentales, Biblioteca de Autores Españoles, vol. 231, Ediciones

[1629] Atlas, Madrid.

Vega Ocampo, A.

1979 Importancia arqueológica de la Provincia de Bolivar, Universidad Nacional de Trujillo, Trujillo.

Werthemann, A.

1892 Ruinas de la fortaleza de Cuelap, Boletín de la Sociedad Geográfica de Lima 2, 4-6.

Wiener, C.

1884 Amazone et cordilleres, Tour du Monde 48, 385-416.

Zovar, J.

2012 Post-Collapse Constructions of Community, Memory, and Identity: an archaeological analysis of Late Intermediate Period community formation in Bolivia's Desaguadero Valley, tesis doctoral, Departamento de Antropología, Vanderbilt University. 